\title{
ANALYSE RÉGIONALE DES SYSTÈMES DISTRIBUÉS
}

\begin{abstract}
A. EL JAI ${ }^{1}$
Abstract. The aim of this paper is to give the state of the art in the regional analysis of distributed parameter systems. The statement of regional analysis problems is: given a dynamical system defined on a domain $\Omega$, one focuses the study of its controllability, its observability, its stability, ... only on a given subregion $\omega, \omega \subset \Omega$. We develop the extension of classical concepts as well as new concepts like spreadability. Various results and examples illustrate the paper.

Résumé. Le but de cet article est de montrer l'état de l'art dans l'analyse des systèmes distribués lorsque l'on examine certains concepts à partir de considérations régionales. Autrement dit, à partir de la donnée d'un système dynamique défini sur un domaine $\Omega$, on ne s'intéresse à sa contrôlabilité, à son observabilité, à sa stabilité, ... que sur une région privilégiée $\omega, \omega \subset \Omega$. Partant de concepts classiques, on développe leur adaptation au cas régional. On développe ensuite des concepts régionaux propres aux systèmes distribués tels que l'étalabilité. Divers développements spécifiques sont donnés. De nombreux résultats et exemples illustrent le travail.
\end{abstract}

Classification Mathématique. 93C20.

Reçu le 14 janvier 2002.

\section{INTRODUCTION}

Un système physique peut être défini brièvement comme un ensemble d'objets (d'entités ou de sous-systèmes) interconnectés et interagissant entre eux. Mathématiquement le système sera représenté par une maquette virtuelle à base d'équations et de signes, c'est-à-dire un modèle. Ce système est dit distribué (ou à paramètres distribués ou encore à paramètres répartis) s'il fait intervenir des variables d'espace et de temps ainsi que des variables d'entrée (commandes) et de sortie (mesures). Ce système est lié à son environnement par l'intermédiaire d'entrées (les sources ou actions ou contrôles, exercés par l'intermédiaire d'actionneurs) et de sorties (les mesures ou observations, utilisant des capteurs).

Traditionnellement l'activité minimale sur un système pour réaliser un certain objectif passait par les étapes de Modélisation $\rightarrow$ Identification $\rightarrow$ Commande. Il est possible aujourd'hui d'enrichir ce schéma par une étape d'analyse qui permet une meilleure compréhension du système et de son fonctionnement et par conséquent de choisir plus justement les méthodes, les algorithmes et même les outils de simulation. On aboutit alors à un

Mots-clés et phrases : Systèmes distribués, contrôlabilité, actionneurs, étalabilité, contrôle.

1 Laboratoire de Théorie des Systèmes - LTS, Université de Perpignan, 52 avenue de Villeneuve, 66860 Perpignan Cedex, France ; e-mail : eljai@univ-perp.fr 
schéma de type Modélisation $\rightarrow$ Analyse $\rightarrow$ Commande. L'analyse est une étape englobant l'étude d'un certain nombre de concepts qui vont permettre d'affiner la démarche vers le choix des actions, par exemple. C'est au niveau de cette étape que se situe ce travail.

Nous pouvons distinguer trois familles de problèmes en relation avec les systèmes distribués.

Les problèmes de Modélisation. La modélisation des systèmes distribués est traditionnellement faite avec des équations aux dérivées partielles. Les mathématiques sous-jacentes ont progressé de façon très significative offrant des cadres d'étude adaptés à de nombreuses situations complexes. Mais la plupart des utilisateurs de modèles, en particulier dans les sciences du vivant, ne sont pas très familiers avec ces avancées théoriques. La faible connection qu'il y a entre les approches par EDP et les utilisateurs de modèles réels est due au jargon mathématique qui leur est peu familier. Or la principale qualité d'un modèle doit être sa simplicité. C'est pourquoi de nombreux utilisateurs s'orientent vers de nouveaux modèles qui restent accessibles et qui permettent de décrire des situations très complexes. Pour cela, les automates cellulaires constituent un très bon candidat pour les systèmes distribués. Introduits depuis les années 40, ils ont repris du service avec les travaux de Wolfram [94]. Aujourd'hui ils sont de très bons représentants pour les systèmes spatio-temporels en général et permettent de décrire des situations réellement complexes. S'ils ont servi jusque là comme simple outils de modélisation, on commence à les introduire comme outils d'analyse, voire bientôt de contrôle. Pour plus de détails dans cette direction, voir $[38,55]$ et $[60,68,74]$. Mais dans ce travail on restera dans le cadre traditionnel d'une représentation par EDP.

Les problèmes d'analyse. L'analyse concerne l'étude d'un certain nombre de concepts qui permettent une meilleure compréhension du système et de son fonctionnement. La difficulté dans l'analyse des systèmes décrits par des équations aux dérivées partielles vient, entre autres, du fait que les concepts, assez simples dans le cas des systèmes localisés, peuvent être formulés et étudiés à divers degrés à cause de la dimension infinie de l'espace d'état. Parmi les notions fondamentales constituant l'analyse des systèmes, notons celles de contrôlabilité, observabilité, stabilité et stabilisabilité, robustesse, détectabilité, identifiabilité, compensation, observateur, etc. À ces notions s'ajoutent de nouveaux concepts liés essentiellement à la présence de la variable spatiale et qui correspondent à de nombreuses situations réelles. Ce sont les concepts d'analyse via capteurs et actionneurs, voir [58], d'analyse régionale au sens large, voir [59,96], de source et de détection de source, voir [1,2,4], ou encore d'étalabilité, voir $[28,30,56,57]$ ou de remédiabilité [6,7].

L'objet de ce travail est de développer deux exemples de concepts régionaux : la contrôlabilité régionale et l'étalabilité.

Les problèmes de dimensionnement et de contrôle. Il s'agit là de divers problèmes concernant la recherche de paramètres géométriques optimaux (liés à la dynamique du système, aux dimensions de son support géométrique, au nombre et support d'actionneurs ou de capteurs) ou encore de stratégies optimales (fonctions dépendant du temps et/ou de l'espace) permettant d'agir sur le système afin d'atteindre certains objectifs (moindre coût, précision maximale, temps minimal, ...). Les problèmes de contrôle trouvent une solution mathématique explicite au moins dans le cadre linéaire quadratique. Dans le cas non linéaire, les approches sont faites au cas par cas ; de nombreux problèmes ont fait l'objet d'une intense activité scientifique. Quant aux travaux liés à la recherche de paramètres optimaux, diverses approches paramètriques et géométriques ont été développées au cas par cas suivant la dépendance du système par rapport à ces paramètres. Cela n'entre pas dans le cadre de ces notes.

\section{UN EXEMPLE DE CONCEPT RÉGIONAL : LA CONTRÔLABILITÉ RÉGIONALE}

La contrôlabilité régionale est présentée ici à titre d'exemple. Des développements similaires ont été faits pour d'autres concepts (observabilité, détectabilité, ...). La contrôlabilité est une notion complètement maîtrisée dans le cas linéaire localisé. Dans le cas des systèmes distribués, elle peut être définie à divers degrés. Supposons qu'un système soit représenté géométriquement dans un domaine $\Omega$ et donnons-nous une partie $\omega$ de $\Omega$. Désignons par $y=y(x, t, u)$ l'état d'un tel système ( $y$ est une fonction de la variable d'espace $x$, du temps $t$ et de la commande $u$ ) et suppososns que $y$ soit dans un espace d'état $X$ ( $X$ faisant intervenir le domaine spatial, $X=L^{2}(\Omega)$ par exemple). Soit, par ailleurs, $U$ l'espace de commande ( $U$ pouvant également faire intervenir 
une variable d'espace puisque la commande peut agir "régionalement"). Notons enfin $T$ l'horizon de temps considéré.

\subsection{Définitions}

1. Contrôlabilité régionale exacte

Le système $(S)$ est dit exactement régionalement contrôlable sur $\omega$ (ou encore exactement $\omega$-contrôlable) si pour tout $y_{d}$ donné dans $L^{2}(\omega)$, il existe un contrôle $u \in U$ tel que

$$
\left.y(., T, u)\right|_{\omega}=y_{d} .
$$

\section{Contrôlabilité régionale faible}

Le système $(S)$ est dit faiblement régionalement contrôlable (ou encore faiblement $\omega$-contrôlable) si pour tout $y_{d} \in X$, et pour toute marge d'erreur $\varepsilon$ donnée, il existe un contrôle $u \in U$ tel que

$$
\left\|\left.y(., T, u)\right|_{\omega}-y_{d}\right\|_{L^{2}(\omega)} \leq \varepsilon
$$

où $\left.y\right|_{\omega}$ est la restriction de $y$ à $\omega$.

Il est à noter immédiatement que, dans le cas distribué, la notion d'exacte contrôlabilité (globale ou regionale) n'est pas adaptée et reste très peu réalisable même pour une action exercée sur tout le domaine $\Omega$; à moins de considérer des espaces d'état très particuliers. C'est pourquoi nous adoptons la notion de faible contrôlabilité.

Remarquons que dans ces définitions, on ne prête pas attention à l'état final du système sur $\Omega \backslash \omega$. Ces notions simples ont donné lieu à de multiples développements. Nous allons étudier, à travers l'exemple, la notion de contrôlabilité régionale et montrer comment l'analyse peut être menée de façon constructive.

Nous allons développer une illustration de ce qui précède en considérant le système parabolique suivant.

$$
\left\{\begin{array}{l}
\frac{\partial y}{\partial t}=\Delta y \quad Q \\
y(x, 0)=y_{0}(x) \Omega \\
y(\xi, t)=0 \quad \Sigma
\end{array}\right.
$$

où $\Omega$ est un ouvert borné de $\mathbb{R}^{n}$, de frontière régulière $\left.\partial \Omega, Q=\Omega \times\right] 0, T[$ et $\Sigma=\partial \Omega \times] 0, T\left[. X=L^{2}(\Omega)\right.$ est considéré, a priori, comme l'espace d'état, mais nous reviendrons sur ce point plus loin. Divers types d'actions peuvent être considérées sur ce système pour illustrer les propos. Rappelons les définitions suivantes.

\subsection{Actionneurs $\omega$-stratégiques}

1. Actionneur

On appelle actionneur tout couple $\left(\Omega_{0}, g_{0}\right)$ où

- $\Omega_{0} \subset \Omega$ est le support géométrique de l'action;

- $g_{0} \in L^{2}\left(\Omega_{0}\right)$ est la répartition spatiale de l'action dans $\Omega_{0}$. Dans le cas d'action ponctuelle exercée en

$b \in \Omega$, on aura $\Omega_{0} \equiv\{b\}$ et $g_{0} \equiv\left\{\delta_{b}\right\}$ (masse de Dirac concentrée en $b$ ), il faudra alors préciser le sens à donner à l'équation (2.1). D'autres types d'actions ont été envisagés.

\section{Actionneur stratégique}

On dit qu'un actionneur est stratégique (respectivement $\omega$-stratégique) si le système qu'il excite est faiblement contrôlable (respectivement faiblement $\omega$-contrôlable) .

On a des résultats sur l'existence de tels actionneurs et une caractérisation générale dans le cas d'un système excité par $p$ actionneurs $\left(\Omega_{i}, g_{i}\right)_{1 \leq i \leq p}$. 
Proposition 2.1. La suite d'actionneurs $\left(\Omega_{i}, g_{i}\right)_{1 \leq i \leq p}$ est faiblement $\omega$-stratégique si et seulement si :

1. $p \geq \sup _{n} r_{n}$

2. rang $G_{n}=r_{n}, \forall n$, où $G_{n}$ est définie par :

$$
\left(G_{n}\right)_{i, j}=<g_{i}, \varphi_{n_{j}}>_{L^{2}\left(\Omega_{i}\right)}
$$

$1 \leq i \leq p$ et $1 \leq j \leq r_{n},\left(\varphi_{n_{j}}\right)$ est un système complet de fonctions propres de $L^{2}(\omega)$ et $\left(\lambda_{n}\right)$ les valeurs propres associées supposées de multiplicité $r_{n}$.

\section{Remarques.}

1. Cette caractérisation permet le choix de paramètres concrets assurant le transfert du système vers des états choisis a priori.

2. Ce résultat s'étend aux cas d'actionneurs de type ponctuels, ou frontières. Pour les démonstrations ainsi que d'autres détails, voir [34-36, 65, 96].

Difficultés. Dans ce qui précède, au moins deux difficultés d'ordre mathématique ou conceptuel sont à souligner.

- Sur le choix de l'espace d'état. Nous avons choisi comme espace d'état $X=L^{2}(\Omega)$. Ce choix est correct compte tenu des profils raisonnables qu'on peut considérer, d'énergie finie. Si, maintenant, l'actionneur $\left(\Omega_{0}, g_{0}\right)$ amène le système vers un état $\tilde{y}$ qui est moins régulier, c'est à dire $\tilde{y} \in Y$ avec $Y \supset X$, alors on a deux possibilités :

1. $Y$ convient comme nouveau choix d'espace d'état;

2. agir sur la régularité du contrôle pour ramener l'état $\tilde{y}$ à $X$ (on compense, en quelque sorte, l'irrégularité résultant du choix des actionneurs par plus de régularité sur le contrôle). À titre d'exemple, considérons deux situations simples qui conduisent à cette difficulté.

Cas 1 : Action zone frontière. Reprenons le système (2.1)

$$
\left\{\begin{array}{l}
\frac{\partial y}{\partial t}=\Delta y \quad Q \\
y(x, 0)=y_{0}(x) \quad \Omega \\
y(\xi, t)=g_{0}(\xi) u(t) \Sigma
\end{array}\right.
$$

qu'on suppose excité par un actionneur zone frontière $\left(\Gamma_{0}, g_{0}\right)$ avec $\Gamma_{0} \subset \partial \Omega$ et $g_{0}=0$ sur $\Gamma \backslash \Gamma_{0}$.

Dans ce cas on obtient une caractérisation identique à celle de la proposition 2.1 si on considère comme espace d'état $X=L^{2}(\Omega)$ pourvu que le contrôle $u \in L^{r}(0, T)$ avec $r>4$. Notons que dans ce cas, ou plus généralement dans le cas de $p$ actionneurs frontières $\left(\Gamma_{i}, g_{i}\right)_{1 \leq i \leq p}$, la condition aux limites sur $\Sigma$ s'écrit : $y(\xi, t)=\sum_{i=1}^{p} g_{i}(\xi) u_{i}(t)$ et la matrice $G_{n}$ définie en $(2.2)$ a pour éléments

$$
\left(G_{n}\right)_{i, j}=\left\langle g_{i}, \frac{\partial \varphi_{n_{j}}}{\partial \nu}\right\rangle_{L^{2}\left(\Gamma_{i}\right)}
$$

Dans le cas d'un contrôle avec condition de Neumann, l'équation (2.4) devient $\left(G_{n}\right)_{i, j}=<g_{i}, \varphi_{n_{j}}>_{L^{2}\left(\Gamma_{i}\right)}$. 
Cas 2 : Action ponctuelle frontière. Si on considère le même système avec une action ponctuelle exercée au point $b \in \partial \Omega$.

$$
\begin{cases}\frac{\partial y}{\partial t}=\Delta y & Q \\ y(x, 0)=y_{0}(x) & \Omega \\ y(\xi, t)=\delta(\xi-b) u(t) \Sigma & \end{cases}
$$

Dans ce cas, on obtient la même caractérisation que dans la proposition 2.1 avec $X=L^{2}(\Omega)$ pourvu que le contrôle $u \in C_{0}^{\infty}(0, T)$. Comme pour le cas zone frontière, et si on a $p$ actionneurs ponctuels localisés en $\left(b_{i}\right)_{1 \leq i \leq p}$, les éléments de la matrice $G_{n}$ définie en $(2.2)$ deviennent :

$$
\left(G_{n}\right)_{i, j}=\frac{\partial \varphi_{n_{j}}\left(b_{i}\right)}{\partial \nu}
$$

Dans le cas d'un contrôle avec condition de Neumann, $\left(G_{n}\right)_{i, j}=\varphi_{n_{j}}\left(b_{i}\right)$. Voir [52-54].

- Sur le nombre d'actionneurs. La caractérisation des actionneurs $\omega$-stratégiques de la proposition 2.1 fait apparaître une condition sur le nombre minimum d'actionneurs permettant d'amener le système vers des états désirés dans $X$. En fait, cette condition peut être relaxée moyennant une faible perturbation de la frontière du domaine $\Omega$. En effet, on montre qu'on peut ramener l'ordre de multiplicité des valeurs propres à $r_{n}=1, \forall n$. Avec cette propriété, la condition 1 de la proposition 2.1 devient $p \geq 1$. Et ainsi, si la deuxième condition (condition de rang) est satisfaite, alors l'actionneur est $\omega$-stratégique. Dans le cas où la dimension d'espace est égale à 2, ce résultat s'illustre très facilement. Pour plus de détails, voir [55,60,79,96].

\subsection{Un exemple}

Il existe des systèmes distribués non contrôlables mais qui sont régionalement contrôlables. Un contreexemple est présenté ci-dessous, d'autres cas sont illustrés dans [58,65,96] et les références qui y sont citées. L'exemple ci-dessous est académique, certes, mais il montre bien que la notion de contrôlabilité régionale peut être satisfaite par un système qui n'est pas contrôlable (au sens habituel). Pour cela on considère le système défini sur $\Omega=] 0,1[$ et décrit par l'équation parabolique

$$
\left\{\begin{array}{lll}
\frac{\partial y}{\partial t}(x, t)-\frac{\partial^{2} y}{\partial x^{2}}(x, t) & =\chi_{[a, b]} u(t) & \\
y(x, 0) & =0 & ] 0,1[\times] 0, T[ \\
y(0, t)=y(1, t) & =0 & ] 0, T[.
\end{array}\right.
$$

C'est un système de diffusion excité par une action localisée sur le sous-domaine $D=[a, b] \subset] 0,1[$ avec une distribution spatiale de l'action égale à 1 sur $D$. Supposons que $a$ et $b$ sont tels que $(b-a) \notin Q$. Nous avons alors le résultat suivant.

Proposition 2.2. Le système (2.5) n'est pas contrôlable (au sens faible) sur ]0,1[ mais il est faiblement $\omega$ contrôlable sur $\omega=] \alpha, \beta[\subset] 0,1[$ pour $\alpha$ et $\beta$ convenablement choisis.

Le système (2.5) peut être mis sous forme d'équation d'état en posant $A=\frac{\partial^{2}}{\partial x^{2}}$ et $B u=\chi_{[a, b]} u$. L'operator $A$ génère un semi-groupe fortement continu $(S(t))_{t \geq 0}$ dans $X=L^{2}(0,1)$ donné par

$$
S(t) z=\sum_{i=1}^{\infty} \mathrm{e}^{\lambda_{i} t}\left\langle z, \varphi_{i}\right\rangle \varphi_{i}
$$


où

$$
\varphi_{i}(x)=\sqrt{2} \sin (i \pi x) \text { et } \lambda_{i}=-i^{2} \pi^{2} .
$$

Finalement (2.5) s'écrit

$$
\dot{z}=A z+B u \quad ; z_{0}=0 .
$$

Pour l'étude de la faible contrôlabilité, il est alors commode de considérer l'opérateur $H: U \rightarrow X$ donné par

$$
H u=\int_{0}^{T} S(T-s) \chi_{[a, b]} u(s) \mathrm{d} s
$$

qui a pour adjoint $H^{\star}$ avec

$$
\left(H^{\star} z\right)(t)=B^{\star} S^{\star}(T-t) z=\sum_{j=1}^{\infty} \mathrm{e}^{\lambda_{j}(T-t)}\left\langle z, \varphi_{j}\right\rangle \int_{a}^{b} \varphi_{i}(x) \mathrm{d} x
$$

où $\langle$,$\rangle désigne le produit scalaire dans L^{2}(0,1)$. Pour $a$ et $b$ tels que $(b-a) \in Q$ le système (2.5) n'est pas contrôlable (faiblement) car alors $\operatorname{Ker} H^{*} \neq\{0\}$ (voir [65]). Montrons alors qu'il existe des états qui ne sont pas atteignables dans $L^{2}(0,1)$ mais qui sont atteignables dans $L^{2}(\omega)$ pour un choix de $\omega \subset(0,1)$ convenable. Notons $\left(\varphi_{j}\right)_{j \in J}$ une base de fonctions propres de $\operatorname{Ker} H^{*}$, alors nous avons

$$
J=\{j / j(b-a) \in 2 N, N \in \mathbb{N}\} \neq \emptyset
$$

puisque $(b-a) \in Q$. Considérons maintenant la région $\omega=[\alpha, \beta] \subset[0,1]$ telle que $\beta=\alpha+(b-a)$. Pour les $i$ et $j \in J$, nous avons

$$
\int_{\alpha}^{\beta} \varphi_{i}(x) \varphi_{j}(x) \mathrm{d} x=\left\{\begin{array}{lll}
\frac{\beta-\alpha}{2} & \text { si } & i=j \\
0 & \text { si } i \neq j .
\end{array}\right.
$$

1. Pour $j_{0} \in J, \varphi_{j_{0}} \in \operatorname{Ker} H^{*}$ et par conséquent $\varphi_{j_{0}} \in L^{2}(0,1)$ n'est pas atteignable.

2. Montrons que $\varphi_{j_{0}}$ est régionalement atteignable pour un choix convenable de $\omega$, c'est-à-dire encore $\chi_{\omega} \varphi_{j_{0}} \notin$ $\operatorname{ker} H^{*} \chi_{\omega}^{*}$. Nous avons

$$
\begin{aligned}
H^{*} \chi_{\omega}^{*}\left(\chi_{\omega} \varphi_{j_{0}}\right) & =\sum_{k=1}^{\infty} \mathrm{e}^{-\lambda_{k}(T-t)}\left\langle\varphi_{j_{0}}, \varphi_{k}\right\rangle_{L^{2}(\omega)} \int_{a}^{b} \varphi_{k}(x) \mathrm{d} x \\
& =\sum_{k \notin J} \mathrm{e}^{-\lambda_{k}(T-t)}\left\langle\varphi_{j_{0}}, \varphi_{k}\right\rangle_{L^{2}(\omega)} \int_{a}^{b} \varphi_{k}(x) \mathrm{d} x .
\end{aligned}
$$

Ceci montre que $H^{*} \chi_{\omega}^{*}\left(\chi_{\omega} \varphi_{j_{0}}\right) \neq 0$ en général, car sinon on aurait $\left\langle\varphi_{j_{0}}, \varphi_{k}\right\rangle_{L^{2}(\omega)}=0 \quad \forall k \notin J$, ce qui donnerait alors si on choisit $k_{0}\left(k_{0} \notin J\right)$ tel que $k_{0}(b-a)=2 k+1$

$$
\left\langle\varphi_{j_{0}}, \varphi_{k_{0}}\right\rangle_{L^{2}(\alpha, \beta)}=0 \Longleftrightarrow k_{0} \tan j_{0} \pi \alpha=j_{0} \tan k_{0} \pi \alpha
$$

ce qui n'est pas vrai en général (considérer, par exemple, $\alpha=\frac{1}{4} ; b-a=\frac{1}{2} ; j_{0}=4, k_{0}=6$ ).

Finalement $\varphi_{j_{0}}$ est regionalement atteignable sur $\omega=[\alpha, \beta]$. 


\subsection{Contrôle réalisant le transfert régional}

Nous allons maintenant reprendre le système (2.1) et donner la forme d'un contrôle réalisant la contrôlabilité régionale. La méthode est une extension de la méthode HUM développée par Lions [77].

$$
\begin{cases}\frac{\partial y}{\partial t}=\Delta y+g_{0}(x) u(t) & Q \\ y(x, 0)=y_{0}(x) & \Omega \\ y(\xi, t)=0 & \Sigma .\end{cases}
$$

Le système (2.9) est excité par un actionneur zone localisé sur $\Omega_{0}$ avec la répartition spatiale $g_{0}$. Considérons une région $\omega \subset \Omega$ et soit $\chi_{\omega}$ la restriction à $\omega$

$$
\chi_{\omega}: L^{2}(\Omega) \longrightarrow L^{2}(\omega)
$$

définie par $\chi_{\omega} y=\left.y\right|_{\omega}$. Imaginons que le problème consiste à ramener au repos le système (2.9) sur la région $\omega$, c'est-à-dire à réaliser : $y(x, T, u)=0 \forall x \in \omega$. Pour résoudre ce problème, nous allons procéder de la façon suivante. L'espace d'état étant $X=L^{2}(\Omega)$, soit

$$
G=\{g \in X \mid g=0 \text { dans } \omega\}
$$

et

$$
G^{0}=\left\{\varphi^{0} \in X \mid \varphi^{0}=0 \text { dans } \Omega \backslash \omega\right\}
$$

Si l'actionneur $\left(\Omega_{0}, g_{0}\right)$ est stratégique alors l'application

$$
\varphi^{0} \longrightarrow\left\|\varphi^{0}\right\|^{2}
$$

avec

$$
\left\|\varphi^{0}\right\|^{2}=\int_{0}^{T}\left\langle\varphi(t), g_{0}\right\rangle_{L^{2}\left(\Omega_{0}\right)}^{2} \mathrm{~d} t
$$

où $\varphi$ est défini par

$$
\begin{cases}\frac{\partial \varphi}{\partial t}(x, t)=\Delta \varphi(x, t) & Q \\ \varphi(x, T)=\varphi^{0}(x) & \Omega \\ \varphi(\xi, t)=0 & \Sigma\end{cases}
$$

définit une norme sur $G^{0}$. Le contrôle $u$ qui ramène le système (2.9) au repos en $T$ sur $\omega$ est donné par :

$$
u(t)=-\left\langle\varphi(t), g_{0}\right\rangle_{L^{2}\left(\Omega_{0}\right)}
$$

où $\varphi$ est solution de l'équation $(2.15)$ avec un état final $\varphi^{0}$ convenable. Pour obtenir $\varphi^{0}$, on considère l'opérateur $\Lambda$ défini par :

$$
\Lambda \varphi^{0}=-\chi_{\omega}^{*} \chi_{\omega}\left(y_{1}(T)\right)
$$


où $\chi^{*}$ est défini de façon naturelle en prolongeant par 0 sur $\Omega \backslash \omega$ et $y_{1}$ est solution de

$$
\begin{cases}\frac{\partial y_{1}}{\partial t}=\Delta y_{1}+g_{0}(x) u(t) & Q \\ y_{1}(x, 0)=0 & \Omega \\ y_{1}(\xi, t)=0 & \Sigma .\end{cases}
$$

On montre que $\Lambda$ est un isomorphisme sous l'hypothèse que l'actionneur $\left(\Omega_{0}, g_{0}\right)$ est stratégique. Finalement l'état $\varphi^{0}$ convenable est solution de l'équation :

$$
\Lambda \varphi^{0}=\chi_{\omega}^{*} \chi_{\omega}\left(y_{0}(T)\right)
$$

où

$$
\begin{cases}\frac{\partial y_{0}}{\partial t}=\Delta y_{0} & Q \\ y_{0}(x, 0)=y_{0}(x) & \Omega \\ y_{0}(\xi, t)=0 & \Sigma .\end{cases}
$$

Pour plus de détails et quelques résultats de simulation, voir $[58,65,96]$.

Remarques. Intérêts de la contrôlabilité régionale.

La notion de contrôlabilité régionale est plus adaptée aux systèmes décrits par des équations aux dérivées partielles. Parmi les raisons de cela :

- c'est un concept plus proche de préoccupations concrètes (maintien d'une température donnée dans une région, ...);

- on montre qu'un transfert régional est à moindre coût si $\omega \nsubseteq \Omega$;

- le transfert régional est plus précis (en ce qui concerne l'objectif à atteindre).

Divers autres concepts liés à l'analyse des systèmes distribués peuvent être étudiés par des approches similaires. De nombreuses illustrations se trouvent dans $[48,62,63,65,66]$ et les références qui y sont citées.

Une extension de divers résultats d'analyse régionale au cas des systèmes distribués discrets a été explorée par Afifi et al. [1,3]. Cette approche des systèmes distribués ouvre un large horizon sur l'utilisation simplifiée et plus raisonnable de l'automatique des systèmes modélisés par des équations aux dérivées partielles. À côté de ces développements, il reste de multiples problèmes ouverts relatifs, en particulier, aux relations entre la région $\omega$ et une "bonne" localisation des actionneurs.

\subsection{Notion de source et de détection de source}

Les problèmes de source et de détection de source constituent des situations particulières d'analyse régionale et trouvent leurs motivations dans de nombreux domaines réels de l'environnement et des risques technologiques ou naturels. Il s'agit de localiser une source et éventuellement son intensité à partir d'informations recueillies par l'intermédiaires d'un certain nombre de capteurs supposés connus. La source peut être de diverses natures et cela rendra sa détection plus ou moins complexe. La source peut être persistante (dans le temps), impulsionnelle (en condition initiale ou durant l'évolution du système) ou encore variable dans l'espace. Nous allons rappeler quelques définitions et donner des résultats dans des cas simples.

On se donne un système $(S)$ d'état $y$ défini sur un domaine $\Omega$ borné de $\mathbb{R}^{n}$ et $T>0$. Le système $(S)$ est augmenté d'une équation de sortie $(E)$ donnant la mesure $z$.

\section{Définition 2.3.}

1. On appelle source tout couple $\left(\mathcal{B}_{t}, g\right)$ où $\mathcal{B}_{t} \subset \Omega, t \in I$, définit le support de la source et $g=g(x, t) \in$ $L^{2}\left(\mathcal{B}_{t}, I\right)$ définit l'intensité de la source à l'instant $t$ en $x \in \mathcal{B}_{t}$. 
2. La source est dite ponctuelle mobile si $\mathcal{B}_{t}=\left\{b_{t}\right\} \in \Omega$.

3. La source est dite ponctuelle fixe si $\mathcal{B}_{t}=\{b\} \in \Omega$.

4. La source est dite persistante quand $\mu(I)>0$; elle est dite impulsionnelle quand $\mu(I)=0$.

L'objet consiste donc à reconstituer une source totalement ou partiellement, c'est-à-dire, par exemple, déterminer la seule localisation ou l'intensité de la source.

Définition 2.4. Supposons que la sortie $(E)$ soit obtenue par utilisation d'un capteur $(D, f)$.

1. La source $\left(\mathcal{B}_{t}, g\right)$ est dite détectable si la connaissance du système $(S)$ et celle de la fonction de sortie $(E)$ permet de déterminer complètement la source.

2. Le capteur $(D, f)$ est dit capteur-espion s'il permet la détection de la source $\left(\mathcal{B}_{t}, g\right)$.

À partir de ces définitions plusieurs travaux relatifs à

- la caractérisation de capteurs-espions ;

- la relation qu'il y a entre capteur stratégique et capteur-espion ;

- la détection en temps minimal ;

ont été développés dans [2-4,6,8]. La notion de compensation spatiale (ou remédiabilité) a également été développée dans $[6,7]$. Il s'agit dans ces travaux de de déterminer des contrôles régionaux qui permettent de neutraliser l'effet d'une perturbation elle aussi régionale.

\section{Un NOUVEAU CONCEPT RÉGIONAL : L'ÉTALABILITÉ}

La notion d'étalabilité caractérise, pour un système dynamique donné, le fait qu'une certaine propriété $\mathcal{P}$, satisfaite dans une région $\omega$ du domaine géométrique $\Omega$ sur lequel est défini le système, s'étend géométriquement (dans un sens à préciser) à tout le domaine $\Omega$ (qu'elle peut couvrir on non). La notion d'étalabilité trouve sa motivation dans de nombreux problèmes réels parmi lesquels les problèmes d'environnement, de distribution de populations ou plus généralement tous les problèmes biogéographiques. Une dynamique de végétation, une nappe polluante, une produit chimique qui diffuse dans un organisme, ... sont quelques exemples de systèmes étalables.

Considérons un système $(S)$ défini dans un domaine $\Omega$ borné régulier de $\mathbb{R}^{n}$ et notons :

- $x$ et $t$ les variables d'espace $(\in \Omega)$ et de temps $\left(\in\left[t_{0}, T\right]\right)$;

- $z_{0}$ l'état initial supposé donné à l'instant initial $t_{0}$;

- $z=z\left(x, t, z_{0}, t_{0}\right)$ l'état du système $(S)$.

Soit maintenant $p$ un profil donné dans $L^{2}(\Omega)$ et considérons les ensembles :

$$
\omega_{t}=\left\{x \in \Omega \quad \mid z\left(x, t, z_{0}, t_{0}\right)=p(x)\right\}
$$

correspondant aux domaines où l'état du système coïncide avec $p$ à l'instant $t$. Cela est apparenté à l'analyse régionale mais avec une région variable dans le temps.

\section{1. Étalabilité exacte}

Définition 3.1. Le système $(S)$ est dit étalable vers $p$, sur l'horizon de temps $\left[t_{0}, T\right]$, si la famille $\omega_{t}$ est croissante, c'est-à-dire $\omega_{t} \subset \omega_{s}$ pour tout $s, t$ tels que $t_{0} \leq t \leq s \leq T$.

Le système est dit nul-étalable si $p=0$.

\section{Remarques.}

1. La définition suppose le maintien de l'état $p$ sur la région $\omega_{t}$ à tout instant $s, s \geq t$; ce qui constitue une certaine difficulté et qui fait que la dynamique du système ne peut pas être quelconque. 
2. La définition peut être plus générale si l'on se donne une propriété $\mathcal{P}$ définié sur l'espace d'état au lieu de l'état $p$. Dans ce cas (3.1) s'écrit

$$
\omega_{t}=\left\{x \in \Omega \quad \mid \mathcal{P} z\left(x, t, z_{0}, t_{0}\right)\right\} .
$$

3. On peut définir, de manière analogue, la notion de resorbabilité lorsque les sous-domaines $\omega_{t}$ sont décroissants.

4. Dans certains problèmes réels, il peut être plus adéquat de considérer, comme définition, la croissance des aires des ensembles $\omega_{t}$; cela sera examiné plus loin.

L'étalabilité dépend, entre autre, du choix de la distribution spatiale initiale dans $\omega_{t_{0}}$. Posons $t_{0}=0$ et considérons le système suivant :

$$
\begin{cases}\frac{\partial z}{\partial t}+\frac{\partial z}{\partial x}=0 & x>0 ; t>0 \\ z(0, t)=0 & t>0 \\ z(x, 0)=z_{0}(x) & x>0\end{cases}
$$

de solution

$$
z(x, t)= \begin{cases}z_{0}(x-t) & \text { si } x \geq t \\ 0 & \text { si } x<t .\end{cases}
$$

S'intéressant au cas de la nulle-étalabilité, nous avons

$$
z(x, t)=0 \Longleftrightarrow\left\{\begin{array}{l}
z_{0}(x-t)=0 \text { si } x \geq t \\
\text { ou } \\
0 \leq x<t
\end{array}\right.
$$

ce qui donne

$$
\omega_{t}=\{x \in] 0, \infty[\mid z(x, t)=0\}=\left[0, t\left[\cup\left\{\left(t+\omega_{0}\right) \cap\right] t,+\infty[\} .\right.\right.
$$

Ainsi pour $\omega_{0} \subset\left[0,+\infty\left[\right.\right.$, on a $\omega_{t}=\left[0, t\left[\cup\left(t+\omega_{0}\right)\right.\right.$. On remarque que $\omega_{t}$ dépend évidemment de $\omega_{0}$ et des conditions aux limites au point 0 .

Cas 1: Si $\omega_{0}$ est réduit à un point $\neq 0, \omega_{0}=\{b\}$ où $b>0$, alors $\omega_{t}=[0, t[\cup\{b+t\}$. Il est clair que dans ce cas le système (3.3) n'est pas étalable à partir de $\omega_{0}=\{b\}$ car pour tout $s \geq t$, on a $[0, t[\subset[0, s[$ mais $\{b+t\}$ n'appartient pas à $\omega_{s}$.

Cas 2: Si $\omega_{0}$ est un intervalle non vide $\omega_{0}=[a, b]$ avec $0<a<b$. Dans ce cas $\omega_{t}=[0, t[\cup[a+t, b+t]$; le système (3.3) n'est donc pas étalable.

Cas 3: Si $\omega_{0}=\emptyset$, dans ce cas, $\omega_{t}=\left[0, t\left[\right.\right.$ et alors le système est nul-étalable, la croissance des domaines $\omega_{t}$ est due à la condition aux limites homogène.

Sans perdre de généralité, on peut supposer que l'état dont on désire l'étalement est $p=0$ (cas de la nulleétalabilité). La caractérisation de l'étabilité va découler de l'inclusion entre les domaines $\omega_{t}$ ou encore, plus simplement, de l'inclusion $\omega_{t} \supset \omega_{t_{0}}, \forall t \in\left[t_{0}, T\right]$. Ainsi nous avons les résultats suivants.

Proposition 3.2. Le système $(S)$ est étalable si et seulement si il existe une fonction $\eta: \Omega \times I \rightarrow \mathbb{R}$ telle que : $\forall x \in \Omega$ et $(s, t) \in I=\left\{(s, t) \in\left[t_{0}, T\right] \times\left[t_{0}, T\right] \mid s \geq t\right\}$, nous avons

$$
z(x, s)=\eta(x, s, t) z(x, t) \quad(\eta(x, t, t)=1) .
$$


Nous avons également la condition nécessaire ci-dessous.

Proposition 3.3. Si le système $(S)$ est étalable et si z est différentiable alors il existe une fonction $b: \Omega \times$ $\left[t_{0}, T\right] \rightarrow \mathbb{R}$ dépendant de l'état z et telle que :

$$
\frac{\partial z}{\partial t}=b z \quad \Omega \times\left[t_{0}, T\right]
$$

Ainsi l'équation (3.7), avec $b=b(x, t, z)$ dépendant de l'état $z$, donne une idée sur la dynamique des systèmes étalables. Ces deux résultats conduisent à la caractérisation suivante :

Proposition 3.4. Le système $(S)$ est étalable si et seulement si il existe une fonction $\xi: \Omega \times\left[t_{0}, T\right] \rightarrow \mathbb{R}$ telle que :

$$
\begin{cases}\frac{\partial(\xi z)}{\partial t}+z^{2}=0 & \text { sur } \Omega \times\left[t_{0}, T\right] \\ \lim _{t \rightarrow T} \xi(x, t) z(x, t)=0 & \text { sur } \Omega\end{cases}
$$

Dans la proposition ci-dessus, on peut utiliser, à la place du terme en $z^{2}$, n'importe quelle fonction positive en $z$. Pour la preuve de ces résultats voir $[56,57]$. La notion d'étalabilité est de façon évidente plus contraignante sur un système (elle est plus forte que la contrôlabilité régionale), d'où l'intérêt d'introduire une notion plus faible.

\section{2. Étalabilité faible}

La notion d'étalabilité telle qu'elle a été introduite dans le paragraphe précédent est forte et donc très restrictive pour décrire de nombreuses situations réelles. En effet cela nécessite la détermination exacte des sous-domaines $\omega_{t}$ où l'état du système suit exactement un profil bien déterminé. C'est pourquoi on l'appelle parfois étalabilité exacte [31]. Dans de nombreuses situations, il est suffisant de considérer des approximations des domaines et/ou de l'état. Cela n'est pas facile car le fait de chercher à approcher simultanément l'état du système et les domaines géométriques n'est pas une opération simple.

Tenant compte de cela, nous avons introduit la notion de faible étalabilité de la manière suivante [92]. D'autres approches sont étudiées dans [16].

Définition 3.5. Soit $\varepsilon>0$. On dit que le système $(S)$ est faiblement étalable avec la tolérance $\varepsilon$ pendant l'intervalle de temps $I=] t_{0}, T$, à partir du domaine initial $\omega_{t_{0}}$ non vide, s'il existe une famille de sous-domaines $\sigma=\left(\sigma_{t}\right)_{t \in I}$ de $\Omega$ vérifiant :

1. $\sigma_{t_{0}} \supseteq \omega_{t_{0}}$;

2. $\sigma_{t} \subset \sigma_{s}$ pour tout $s, t$ tels que $t_{0} \leq s \leq t \leq T$;

3. $\sigma_{T}=\Omega$;

4. $\left\|\chi_{\sigma_{t}}(.)(z(., t)-p(.))\right\|_{L^{2}\left(\sigma_{t}\right)} \leq \varepsilon \operatorname{mes}\left(\sigma_{t}\right) \forall t \in I$

où mes $\left(\sigma_{t}\right)$ est la mesure de Lebesgue de $\sigma_{t}$ et $\chi_{\sigma_{t}}$ est la fonction caractéristique de $\sigma_{t}$. On dira que la famille $\sigma=\left(\sigma_{t}\right)_{t \in I}$ de $\Omega$ est un étalement si elle vérifie 1 et 2 .

On notera $S$ l'ensemble des étalements

$$
S=\left\{\sigma=\left(\sigma_{t}\right)_{t \in I} \text { tels que }\left(\sigma_{t}\right) \nearrow \text { et } \sigma_{t_{0}} \supset \omega_{t_{0}}\right\}
$$

et

$$
S^{\prime}=\left\{\sigma \in S \text { tels que } \sigma_{T}=\Omega\right\}
$$




\section{Remarques.}

1. Un système $(S)$ étalable est faiblement étalable pour toute tolérance $\varepsilon$ si à l'instant final $T$, tout le domaine $\Omega$ est couvert. En effet dans ce cas il suffit de choisir $\sigma_{t}=\omega_{t}$.

2. Tout système faiblement $\mathcal{P}$-étalable avec la tolérance $\varepsilon$ est faiblement $\mathcal{P}$-étalable avec la tolérance $\varepsilon^{\prime}$ pour tout $\varepsilon^{\prime} \geq \varepsilon$.

3. Pour tout système dynamique, dont l'état est assez régulier, il existe toujours un $\varepsilon$ positif (dépendant évidemment de l'état du système et du profil $p$ ) pour lequel ce système est faiblement étalable. En effet il suffit de prendre une tolérance $\varepsilon$ supérieure à :

$$
\sup _{t \in I}\left(\frac{\left\|\chi_{\sigma_{t}}(.) \cdot(z(., t)-p(.))\right\|_{L^{2}\left(\sigma_{t}\right)}}{\operatorname{mes}\left(\sigma_{t}\right)}\right)
$$

quand cette quantité est finie.

Les remarques précédentes montrent l'importance de la tolérance $\varepsilon$ et du choix de la famille $\left(\sigma_{t}\right)$. Montrer qu'un système est faiblement étalable revient à trouver des sous-domaines croissants dans le temps (au sens de l'inclusion), où l'état du système est égal à $p$, à $\varepsilon$ près. La détermination de ces sous-domaines croissants, qui reste un problème central dans la notion de faible étalabilité, est facilitée par l'utilisation de l'ensemble $S^{\prime}$. Dans le cas où $p=0$, on a le résultat suivant.

Proposition 3.6. Un étalement $\sigma=\left(\sigma_{t}\right)_{t \in I}$ est tel que $\sigma \in S^{\prime}$ si et seulement si

$$
\sigma_{t}=\{x \in \Omega / \tau(x) \leq t\} \text { pour tout } t \in I
$$

ò̀ $\tau \in \mathcal{T}=\left\{\tau \in L^{2}(\Omega) / 0 \leq \tau(x) \leq T\right.$ et $\left.\left.\right|_{\omega_{0}}=0\right\}$.

Pour la preuve de ce résultat, voir [74].

Remarque 3.7. La proposition 3.6 nous permet de caractériser les étalements $\sigma_{t}$ à l'aide des fonctions mesurables $\tau$ dont la manipulation est plus pratique.

La notion de faible étalabilité permet de considérer, avec une certaine tolérance $\varepsilon$, des étalements $\left(\sigma_{t}\right)$ où l'état du système suit approximativement un profil désiré. Ce qui permet de décrire certains phénomènes dans lesquels on ne peut pas connaître exactement l'état du système ou les domaines $\left(\omega_{t}\right)$.

\section{3. Étalabilité au sens des aires ( $\mathcal{A}$-étalabilité)}

\subsubsection{Limitations de la notion d'étalabilité exacte}

L'étalabilité d'un système dynamique dépend naturellement de la dynamique du système, des données initiales, des actions, etc. Dans la définition de l'étalabilité, l'état coïncide avec le profil $p$ sur les $\left(\omega_{t}\right)$ mais pour $s \geq t$, il y a maintien de l'état (il reste égal à $p$ ). Ce qui n'est pas le cas pour de nombreuses situations. Par exemple, pour un problème de dynamique de végétation (à une echelle de paysage), généralement il y a expansion mais aussi un recouvrement différent (déplacé) du couvert végétal ; ce qui n'est pas décrit par la notion d'étalabilité (au sens de l'inclusion) ni la faible étalabilité si les zones où on "perd" le profil $p$ sont de mesure importante.

\subsubsection{Définition}

Dans les définitions qui suivent, nous supposons que les domaines $\omega_{t}$ sont toujours définis par

$$
\omega_{t}=\left\{x \in \Omega \quad \mid \quad z\left(x, t, z_{0}, t_{0}\right)=p(x)\right\}
$$

à partir de la donnée du profil $p$. 
Définition 3.8. On dit que le système $(S)$ est étalable au sens des aires ou $\mathcal{A}$-étalable pendant l'intervalle de temps $I=\left[t_{0}, T\right]$, si

$$
\operatorname{mes}\left(\omega_{s} \backslash \omega_{t}\right) \geq \operatorname{mes}\left(\omega_{t} \backslash \omega_{s}\right) \forall t, s, \quad t_{0} \leq t \leq s \leq T
$$

La définition ci-dessus signifie simplement que la surface "gagnée" lors de l'étalement est supérieure à celle "perdue" pendant l'intervalle de temps $[t, s], s \geq t$.

Si dans la définition ci-dessus on a mes $\left(\omega_{s} \backslash \omega_{t}\right)>\operatorname{mes}\left(\omega_{t} \backslash \omega_{s}\right) \forall s \geq t \geq t_{0}$, on dira que ce système $(S)$ est strictement étalable au sens des aires.

Si mes $\left(\omega_{s} \backslash \omega_{t}\right) \leq \operatorname{mes}\left(\omega_{t} \backslash \omega_{s}\right) \forall s \geq t$; (respectivement mes $\left.\left(\omega_{s} \backslash \omega_{t}\right)<\operatorname{mes}\left(\omega_{t} \backslash \omega_{s}\right) \forall s \geq t\right)$; on dira que le système $(S)$ est résorbable (respectivement strictement résorbable) au sens des aires.
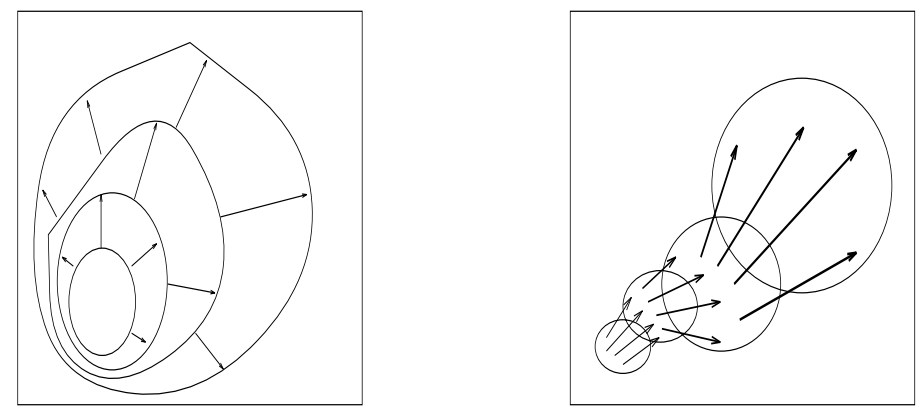

FigurE 1. Étalabilité au sens de l'inclusion et étalabilité au sens des aires.

À partir de ce qui précède, il est facile de noter que si un système $(S)$ est étalable au sens de l'inclusion pendant l'intervalle de temps $I=\left[t_{0}, T\right]$, alors $(S)$ est étalable au sens des aires pendant le même intervalle de temps $I$. La réciproque est évidemment fausse comme le montre l'exemple suivant. Pour $a>0$ suffisamment grand et $\Omega=] 0, a[$, le système décrit par l'équation

$$
\left\{\begin{array}{l}
\frac{\partial z}{\partial t}(x, t)=b(x, t) z(x, t) \quad t>0, x \in \Omega \\
z(x, 0)=z_{0}(x)=h(x+1)
\end{array}\right.
$$

où

$$
b(x, t)= \begin{cases}\frac{x+1}{(t-x)^{2}} & \text { si } 0<\frac{x+1}{t+1}<1 \\ 0 & \text { si } 1 \leq \frac{x+1}{t+1} \leq 2 \\ -\frac{x+1}{(2 t-x+1)^{2}} & \text { si } \frac{x+1}{t+1}>2\end{cases}
$$

et

$$
h(x)= \begin{cases}\exp \left(-\frac{1}{1-x}\right) & \text { si } 0<x<1 \\ 0 & \text { si } 1 \leq x \leq 2 \\ \exp \left(\frac{1}{2-x}\right) & \text { si } x>2\end{cases}
$$


a pour solution

$$
z(x, t)= \begin{cases}\exp \left(-\frac{1}{1-\frac{x+1}{t+1}}\right) & \text { si } 0<\frac{x+1}{t+1}<1 ; t>0 ; x<a \\ 0 & \text { si } 1 \leq \frac{x+1}{t+1} \leq 2 ; t>0 ; x<a \\ \exp \left(\frac{1}{2-\frac{x+1}{t+1}}\right) & \text { si } 2<\frac{x+1}{t+1} ; t>0, x<a .\end{cases}
$$

Considérons $\omega_{0}=[0,1]$ et $\omega_{t}=\{x \in \Omega \mid z(x, t)=0\}$ alors on a, dans ce cas,

$$
\omega_{t}=\left\{x \in \Omega \mid 1 \leq \frac{x+1}{t+1} \leq 2\right\}=[t, 2 t+1] \text { pour } t \leq \frac{a-1}{2} .
$$

Il s'en suit que mes $\left(\omega_{t}\right)=t+1$, le système (3.11) est donc étalable au sens des aires à partir de $\omega_{0}$ mais non étalable au sens de l'inclusion, voir figure 2.

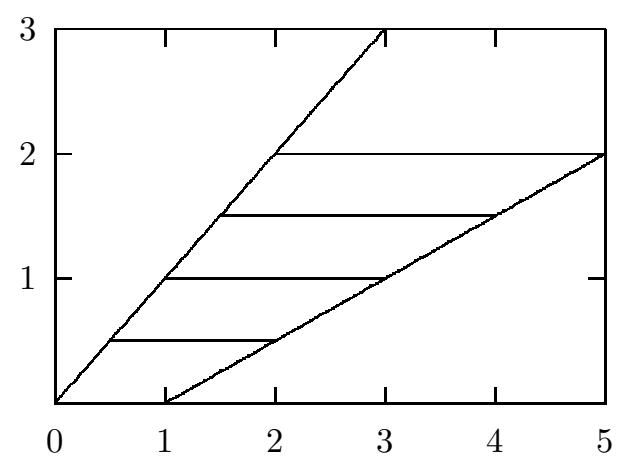

Figure 2. Système étalable au sens des aires mais non étalable au sens de l'inclusion.

Si, dans la définition 3.8, on suppose que $\operatorname{mes}(\Omega)<+\infty$, alors le système est $\mathcal{A}$-étalable si et seulement si

$$
\operatorname{mes}\left(\omega_{s}\right) \geq \operatorname{mes}\left(\omega_{t}\right), \forall s, t \in I \quad s \geq t .
$$

En effet mes $\left(\omega_{s}\right)=\operatorname{mes}\left(\omega_{s} \backslash \omega_{t}\right)+\operatorname{mes}\left(\omega_{s} \cap \omega_{t}\right)$ et mes $\left(\omega_{t}\right)=\operatorname{mes}\left(\omega_{t} \backslash \omega_{s}\right)+\operatorname{mes}\left(\omega_{s} \cap \omega_{t}\right)$, d'où mes $\left(\omega_{s}\right)-$ $\operatorname{mes}\left(\omega_{t}\right)=\operatorname{mes}\left(\omega_{s} \backslash \omega_{t}\right)-\operatorname{mes}\left(\omega_{t} \backslash \omega_{s}\right)$. Par contre, dans le cas où mes $(\Omega)=+\infty$, on n'a pas l'équivalence, voir $[20]$.

Dans cette définition, nous n'avons supposé aucune condition sur le domaine initial $\omega_{t_{0}}$. La condition mes $\left(\omega_{t_{0}}\right) \neq 0$ n'est pas nécessaire mais elle paraît comme naturelle et satisfaite par la plupart des systèmes. Posons $A=\left(\lim _{t \longrightarrow t_{0}^{+}} \omega_{t}^{\prime}\right)$ avec $\omega_{t}^{\prime}=\omega_{t} \backslash A_{t}$ où $A_{t}$ est un ensemble négligeable vérifiant

$$
\bar{A}_{t} \cap \bar{\omega}_{t}=\emptyset \quad\left(\text { i.e. } \inf _{\substack{x \in \omega_{t} \\ y \in A_{t}}} d(x, y)>0\right)
$$

et

$$
\lim _{t \rightarrow t_{0}^{+}} \omega_{t}^{\prime}=B \Longleftrightarrow \lim _{t \rightarrow t_{0}^{+}} h\left(\omega_{t}^{\prime}, B\right)=0
$$


avec $h\left(\omega_{t}^{\prime}, B\right)=\max \left[\rho\left(\omega_{t}^{\prime}, B\right), \rho\left(B, \omega_{t}^{\prime}\right)\right]$, où $h\left(\omega_{t}^{\prime}, B\right)$ est la distance de Hausdorff de $\omega_{t}^{\prime}$ à $B$ et $\rho\left(\omega_{t}^{\prime}, B\right)=$ $\sup d(x, B)$.

$x \in \omega_{t}^{\prime}$

Finalement il peut arriver que, partant d'un ensemble $\omega_{t_{0}}$ de mesure nulle à l'instant $t_{0}$, on obtienne des ensembles $\omega_{t}$, pour $t>t_{0}$, de mesure non nulle. L'ensemble $\lim _{t \rightarrow t_{0}^{+}} \omega_{t}^{\prime}$ peut être interprété comme l'ensemble des points potentiellement étalables (ou points actifs), c'est-à-dire engendrant l'étalabilité. Illustrons cela en reprenant l'exemple (3.3)

$$
\begin{cases}\frac{\partial z}{\partial t}+\frac{\partial z}{\partial x}=0 & x>0 ; t>0 \\ z(0, t)=0 & t>0 \\ z(x, 0)=z_{0}(x) & x>0\end{cases}
$$

Nous avons vu que $\omega_{t}=\left[0, t\left[\cup\left(t+\omega_{0}\right)\right.\right.$, ainsi pour tout $\omega_{0} \subset \mathbb{R}^{+}$, on a mes $\left(\omega_{t}\right)=t$, qui est croissante, donc le système est $\mathcal{A}$-étalable pour tout choix de $\omega_{0}$. Cependant considérons quelques cas particuliers en fonction de la nature du domaine initial et de l'action exercée sur le système :

Cas 1. mes $\left(\omega_{0}\right)>0$, soit par exemple $\omega_{0}=[a, b]$ avec $a<b$, on a vu que $(S)$ est étalable au sens des aires et

$$
\omega_{t}=[0, t] \cup[a+t, b+t] .
$$

L'intervalle $[a+t, b+t]$ se déduit de $[a, b]$ et $[0, t[$ du point 0 (point potentiellement étalable) qui n'est pas forcément dans $\omega_{0}$. En effet, dans ce cas on a :

$$
\lim _{t \rightarrow 0^{+}} \omega_{t}^{\prime}=\{0\} \cup[a, b] .
$$

Cas 2. $\operatorname{mes}\left(\omega_{0}\right)=0$, soit par exemple $\omega_{0}=\{b\}$ avec $b>0$. Dans ce cas nous avons

$$
\omega_{t}=\left[0, t\left[\cup\{b+t\} \quad \text { et } \quad \operatorname{mes}\left(\omega_{t}\right)=t\right.\right.
$$

L'étalabilité est engendrée en quelque sorte par la conditions aux limites homogène.

Pour caractériser l'étalabilité au sens des aires, on considère un système dynamique $(S)$ avec les mêmes hypothèses que dans ce qui précède. Sans perte de généralité, on suppose que mes $(\Omega)<+\infty$ et on ne considérera que le cas de la nulle-étalabilité. Pour étudier la variation de la mesure des domaines $\left(\omega_{t}\right)$, nous allons introduire des familles de transformations permettant de décrire ces domaines.

Définition 3.9. On dit qu'une famille de transformations $(F(., s, t))_{s \geq t}, F: \Omega \times I \times I \rightarrow \Omega$, est adaptée à l'étalabilité du système $(S)$ (ou tout simplement adaptée au système $(S)$ ) s'il existe une fonction $\eta(x, s, t)$, $\eta: \Omega \times I \times I \rightarrow \mathbb{R}$, satisfaisant l'équation

$$
z[F(x, s, t), s]=\eta(x, s, t) z(x, t) .
$$

\section{Remarque 3.10.}

1. Notons qu'une telle famille de transformations permet de décrire l'évolution géométrique des domaines $\omega_{t}$. En effet si $\eta(x, s, t)$ est à valeurs dans $\mathbb{R}^{\star}$, alors $F\left(\omega_{t}, s, t\right)=\omega_{s}$ et si $\eta(x, s, t)$ est à valeurs dans $\mathbb{R}$, alors $F\left(\omega_{t}, s, t\right) \subset \omega_{s}$. 
2. Par ailleurs, si une famille de transformations est adaptée à un système $(S)$, elle n'est pas forcément unique. En effet, considérons toujours l'exemple donné par l'équation (3.11), alors les familles de transformations

$$
F_{1}(x, s, t)= \begin{cases}\frac{s+1}{t+1} x+s-\frac{s+1}{t+1} t & \text { si } x \in[t, 2 t+1] \\ \frac{2 s+1}{2 t+1} x & \text { si } x>2 t+1\end{cases}
$$

et

$$
F_{2}(x, s, t)=\left\{\begin{array}{lc}
\frac{s+1}{3 t^{2}+4 t+1} x^{2}+\left[s-\frac{s+1}{\left(3 t^{2}+4 t+1\right)} t^{2}\right] & \text { si } x \in[t, 2 t+1] \\
\frac{2 s+1}{2 t+1} x & \text { si } x>2 t+1
\end{array}\right.
$$

sont deux familles adaptées à ce système. Donc on peut parler de classe de familles de transformations adaptées à un système.

Nous avons la caractérisation suivante :

Proposition 3.11. Une famille de transformations $(F(., s, t))_{s \geq t}$ est adaptée au système $(S)$ si et seulement si il existe une fonction $\xi(x, t)$ solution de l'équation

$$
\left\{\begin{array}{l}
\frac{\partial}{\partial t}(\xi(x, t) z(x, t))+\frac{\partial H}{\partial t}(x, t)=0 \\
\xi(x, T) z(x, T)=0
\end{array}\right.
$$

où $H(x, t)=\int_{t}^{T} z^{2}[F(x, s, t), s] \mathrm{d} s$.

Démonstration. Il suffit de noter que (3.20) est équivalente à

$$
\xi(x, t) z(x, t)=\int_{t}^{T} z^{2}[F(x, s, t), s] \mathrm{d} s
$$

d'où

$z(x, t)=0 \Rightarrow \int_{t}^{T} z^{2}[F(x, s, t), s] \mathrm{d} s=0$ et alors $z[F(x, s, t), s]=0, \forall s \geq t$, d'où $F(x, s, t) \in \omega_{s}$.

Réciproquement si la famille $(F(., s, t))_{s \geq t}$ est adaptée au système $(S)$, il suffit de prendre

$$
\xi(x, t)= \begin{cases}\frac{\int_{t}^{T} z^{2}[F(x, s, t), s] \mathrm{d} s}{z(x, t)} & \text { si } x \in \omega_{t} \\ \text { constante } & \text { sinon. }\end{cases}
$$

Ce résultat conduit à la caractérisation suivante de l'étalabilité. 
Proposition 3.12. Un système $(S)$ est $\mathcal{A}$-étalable si et seulement si il existe une famille de transformations $(F(., s, t))_{s \geq t}$ adaptée à $(S)$ et vérifiant mes $\left(F\left(\omega_{t}, s, t\right)\right) \geq \operatorname{mes}\left(\omega_{t}\right)$ pour tout $s \geq t \geq t_{0}$.

Si la famille de transformations $(F(., s, t))_{s \geq t}$ de $\Omega$ est une famille de difféomorphismes de $\Omega$, alors le système $(S)$ est étalable au sens des aires si et seulement si

$$
\int_{\omega_{t}}[|\mathcal{J} F(x, s, t)|-1] \mathrm{d} x \geq 0 ; \quad \forall s, t, s \geq t \geq t_{0}
$$

ou, de façon équivalente, si $F\left(\omega_{t}, s, t\right)=\omega_{s} \forall s, t, s \geq t$,

$$
\frac{\partial}{\partial t} \int_{\omega_{t_{0}}}\left|\mathcal{J} F\left(x, t, t_{0}\right)\right| \mathrm{d} x \geq 0 ; \quad \forall t \geq t_{0}
$$

où $\mathcal{J} F(x, s, t)$ est le jacobien de $F(x, s, t)$ au point $x$.

Démonstration. Posons $F\left(x_{t}, s, t\right)=x_{s}$, si $F\left(\omega_{t}, s, t\right)=\omega_{s}$, alors

$$
\operatorname{mes}\left(\omega_{s}\right)=\int_{\omega_{s}} \mathrm{~d} x_{s}=\int_{\omega_{t}}\left|\mathcal{J} F\left(x_{t}, s, t\right)\right| \mathrm{d} x_{t}
$$

par changement de variable, on a une transformation du domaine qui donne

$$
\int_{\omega_{s}} \mathrm{~d} x_{s} \geq \int_{\omega_{t}} \mathrm{~d} x_{t} \Longleftrightarrow \int_{\omega_{t}}\left[\left|\mathcal{J} F\left(x_{t}, s, t\right)\right|-1\right] \mathrm{d} x_{t} \geq 0 .
$$

De même, et par un raisonnement analogue, on montre que mes $\left(\omega_{t}\right)$ croissante est équivalent à

$$
\frac{\partial}{\partial t} \int_{\omega_{t_{0}}}\left|\mathcal{J} F\left(x_{t_{0}}, t, t_{0}\right)\right| \mathrm{d} x_{t_{0}} \geq 0
$$

Si $F\left(\omega_{t}, s, t\right) \subset \omega_{s}$, et si (3.21) est vérifiée, alors $(S)$ est étalable au sens des aires. En effet

$$
\int_{\omega_{s}} \mathrm{~d} x \geq \int_{F\left(\omega_{t}, s, t\right)} \mathrm{d} x=\int_{\omega_{t}}|\mathcal{J} F(x, s, t)| \mathrm{d} x .
$$

Remarque 3.13. En prenant $F(., s, t)=I d_{\Omega}$ (injection canonique de $\omega_{t}$ dans $\omega_{s}$ ), on retrouve le problème de l'étalabilité au sens de l'inclusion. Ainsi, dans ce cas $(S)$ sera étalable si et seulement si $\left(I d_{\Omega}\right)$ est adaptée à $(S)$, i.e. (3.17) admet une solution $\eta(x, s, t)$ à valeurs dans $\mathbb{R}$; si cette solution est à valeurs dans $\mathbb{R}^{\star}$, alors on a étalabilité au sens large : $\omega_{t}=\omega_{s}$. Notons que, dans ce cas, la condition (3.21) (ou (3.22)) est vérifiée puisque

$$
|\mathcal{J} F(., s, t)|=1 \quad \text { sur } \Omega
$$

et en prenant $(F(., s, t))_{s \geq t}$ tel que $\int_{\omega_{t}}\left[\left|\mathcal{J} F\left(x_{t}, s, t\right)\right|-1\right] \mathrm{d} x_{t} \geq 0$ on a un étalement au sens des aires. Le choix de la famille $(F(., s, t))_{s \geq t}$ est donc important.

\section{4. Étalabilité et ensembles de niveau}

L'objet de ce paragraphe est de voir si nous pouvons décrire les domaines $\left(\omega_{t}\right)$ à partir de l'évolution de leurs frontières $\left(\partial \omega_{t}\right)$. Cela est possible si on a d'autres informations (telle la vitesse d'évolution) sur la manière dont évolue cette frontière. Nous considérons le cas où $\Omega \subset \mathbb{R}^{n}$, avec $n=2$ ou 3 et nous allons voir certaines relations entre la $\mathcal{A}$-étalabilité et l'évolution des $\left(\partial \omega_{t}\right)$ en utilisant les techniques des ensembles de niveau. 


\subsubsection{Principe des ensembles de niveau}

Une interface peut être définie comme étant une région qui divise un domaine en deux (ou plusieurs) sousdomaines dans lesquelles l'état du système satisfait des propriétés différentes. Dans le cas de l'étalabilité, les $\left(\partial \omega_{t}\right)$ vont jouer le rôle d'une interface en évolution. En général, dans les problèmes d'évolution d'interfaces, la vitesse d'évolution est supposée connue. Sethian 1996 [86] a signalé que cette vitesse d'évolution $v=v(L, G, I)$ dépend essentiellement de trois paramètres. $L$ est lié aux propriétés locales de l'interface, $G$ dépend de la géométrie locale (courbure) et des propriétés globales (description différentielle du front, dynamique du système,...) et $I$ est lié à d'autres propriétés indépendantes de l'interface (transport par exemple). Le cas particulier où cette vitesse d'évolution ne dépend que des propriétés locales de l'interface (courbure locale) a été largement étudié par Sethian [86] et par Osher [84] en utilisant les techniques d'ensembles de niveau. Sethian a donné un modèle d'évolution du front dans le cas où la vitesse d'évolution ne dépend que de la courbure locale. Une formulation stationnaire a été établie dans le cas particulier où cette vitesse est positive. Aussi l'équation du modèle a été augmentée par une condition d'entropie justifiée par des considérations physiques. D'autres cas ont fait l'objet de plusieurs études [13, 14,70,89].

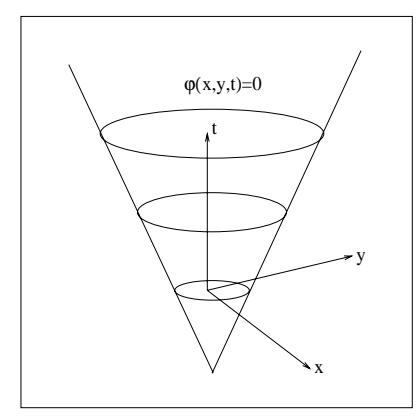

Figure 3. Principe d'ensembles de niveau.

Considérons une hypersurface $\Gamma(t)$ de dimension $(n-1)$ évoluant dans la direction normale avec une vitesse $v=v(\kappa)$ qui dépend uniquement du rayon de courbure locale $\kappa$, à partir d'une position initiale $\Gamma(0)$. Le principe des techniques d'ensembles de niveau consiste à déterminer l'interface $\Gamma(t)$ comme étant l'ensemble de niveau zéro d'une certaine fonction $\varphi=\varphi(x, t)$ de dimension supérieure, voir figure 3 . Cette fonction $\varphi=\varphi(x, t)$, de $n+1$ variables, $x \in \mathbb{R}^{n}$ et $t \in I$, est définie par

$$
\varphi(x, t)= \pm d
$$

où $d$ est la distance du point $x$ à l'interface $\Gamma(t)$; le signe \pm sert à préciser si le point $x$ est intérieur ou extérieur à cette interface. Il s'agit alors de décrire l'évolution de l'interface $\Gamma(t)$ à l'aide de l'ensemble de niveau zéro de $\varphi$. Le but est de trouver une équation en $\varphi(x, t)$ qui décrit l'évolution de $\Gamma(t)$. Pour cela, on détermine d'abord une condition initiale $\varphi(x, 0)$, à partir de $\Gamma(0)$ comme suit

$$
\varphi(x, 0)= \pm d
$$

où $d$ est la distance du point $x$ à $\Gamma(0)$. Ce qui donne $\Gamma(0)=\{x / \varphi(x, 0)=0\}$. Ensuite, comme l'ensemble de niveau zéro de la fonction $\varphi(x, t)$ représente l'interface $\Gamma(t)$ lors de l'évolution, qu'on note $\varphi(x(t), t)=0$, alors en dérivant par rapport à $t$, on obtient [84]

$$
\left\{\begin{aligned}
\varphi_{t}+v|\nabla \varphi|= & 0 \\
\varphi(x, 0) & \text { donnée }
\end{aligned}\right.
$$


avec $x^{\prime}(t) \cdot \vec{n}=v$ et $\vec{n}=\nabla \varphi /|\nabla \varphi|$. Cette équation se réduit, pour certaines formes particulières de $v$, à l'équation de Hamilton-Jacobi standard. Un autre problème important étudié par Sethian [86] concerne l'évaluation de l'étirement total du front, $\operatorname{Var}(t)$. Dans le cas bidimensionnel, si la vitesse d'évolution ne dépend que de la courbure du front $v=v(\kappa)$ où $\kappa$ est la courbure locale du front, par une paramétrisation de $\Gamma(t)$ à l'instant $t$ sous la forme $(x(s, t), y(s, t)), 0 \leq s \leq \alpha$, avec des conditions aux limites périodiques $(x(0, t), y(0, t))=(x(\alpha, t), y(\alpha, t))$, l'étirement total est donné par

$$
\operatorname{Var}(t)=\int_{0}^{\alpha}|\kappa(s, t)|\left(x_{s}^{2}+y_{s}^{2}\right)^{1 / 2} \mathrm{~d} s
$$

où $\kappa=\frac{y_{s s} x_{s}-x_{s s} y_{s}}{\left(x_{s}^{2}+y_{s}^{2}\right)^{3 / 2}}$ est la courbure locale. Pour plus de détails, voir $[85,86]$.

\subsection{2. Étalabilité et ensembles de niveau}

Les techniques d'ensembles de niveau permettent d'étudier les problèmes d'évolution d'interfaces [11, 13,86] alors que la notion d'étalabilité vise à suivre l'évolution des domaines $\left(\omega_{t}\right)$ où une certaine propriété $\mathcal{P}$ donnée est satisfaite. Dans certains cas particuliers, nous pouvons faire le lien entre l'évolution des interfaces $\left(\partial \omega_{t}\right)$ et l'étalabilité. Plaçons-nous dans le cas bidimensionnel et considérons un système $(S)$ défini dans un domaine $\Omega$ et $\omega_{t}=\left\{x \in \Omega \subset \mathbb{R}^{2} \mid \mathcal{P}\right.$ est satisfaite $\}$. Notons $\Gamma_{t}=\partial \omega_{t}$ et supposons que la vitesse $v$ d'évolution de $\Gamma(t)$ dépend uniquement de la courbure $\kappa$ et considérons une paramétrisation de $\Gamma(t)$ sous la forme

$$
\Gamma(t)=\left(\begin{array}{l}
x(s, t) \\
y(s, t)
\end{array}\right) ; 0 \leq s \leq S
$$

En explicitant $x_{t}$ et $y_{t}$ en fonction de la courbure $\kappa$ et la vitesse $v(\kappa)$, on obtient [86]

$$
\left\{\begin{array}{l}
x_{t}=v(\kappa) \frac{y_{s}}{\left(x_{s}^{2}+y_{s}^{2}\right)^{1 / 2}} \\
y_{t}=-v(\kappa) \frac{x_{s}}{\left(x_{s}^{2}+y_{s}^{2}\right)^{1 / 2}}
\end{array}\right.
$$

où

$$
\kappa=\kappa(s, t)=\frac{y_{s s} x_{s}-x_{s s} y_{s}}{\left(x_{s}^{2}+y_{s}^{2}\right)^{3 / 2}}
$$

nous avons alors le résultat suivant.

Proposition 3.14. Supposons que $\Gamma(t)$ est assez régulière et que $x(s, t)$ et $y(s, t)$ admettent des dérivées partielles continues. Notons $\mathcal{V}(t)$ la variation de la mesure des domaines $\left(\omega_{t}\right)$, alors nous avons

$$
\begin{aligned}
\mathcal{V}(t) & =\frac{\mathrm{d} \operatorname{mes}\left(\omega_{t}\right)}{\mathrm{d} t} \\
& =\frac{1}{2} \int_{0}^{S}\left[v(\kappa)\left(x_{s}^{2}+y_{s}^{2}\right)^{1 / 2}-\kappa_{s} v_{\kappa}(\kappa) \frac{x x_{s}+y y_{s}}{\sqrt{x_{s}^{2}+y_{s}^{2}}}-\kappa v(\kappa)\left(y x_{s}-x y_{s}\right)\right] \mathrm{d} s
\end{aligned}
$$

et le système $(S)$ est $\mathcal{A}$-étalable si et seulement si, pour tout $t \in I$

$$
\mathcal{V}(t) \geq 0
$$


Pour la preuve, voir [20]. Dans le concept de la $\mathcal{A}$-étalabilité, la difficulté vient du fait que la vitesse est générée par la dynamique interne du système. Si pour une dynamique donnée, nous pouvons déterminer la vitesse d'évolution de l'interface, alors nous pouvons appliquer ces techniques. Un cas particulier est celui où le front évolue avec une vitesse de propagation constante. Nous considérons le cas bidimensionnel $\left(\Omega \subset \mathbb{R}^{2}\right)$ où la vitesse $v(\kappa)$ est une constante (indépendante de $\kappa)$. Dans ce cas, en posant $x(s, t)=\rho(s, t) \cos s$ et $y(s, t)=\rho(s, t) \sin s$, la relation $(3.29)$ donne

$$
\mathcal{V}(t)=\frac{1}{2} \int_{0}^{S} v(\kappa)\left[\sqrt{\rho^{2}+\rho_{s}^{2}}+\kappa \rho^{2}\right] \mathrm{d} s
$$

ainsi le système est $\mathcal{A}$-étalable si

$$
\int_{0}^{S} v(\kappa)\left[\sqrt{\rho^{2}+\rho_{s}^{2}}+\kappa \rho^{2}\right] \mathrm{d} s \geq 0 \forall t \in I
$$

laquelle condition est satisfaite si $v(\kappa) \geq 0$. C'est le cas aussi si la courbure est constante en tout point $(x(s, t), y(s, t))$; c'est-à-dire si $\kappa_{s}=0$. Ainsi nous pouvons déduire la nature du système ( $\mathcal{A}$-étalable ou pas) sans toutefois chercher la forme du front. Pour la caractérisation de ce dernier et pour plus de détails voir [86].

Dans ce qui précède, nous avons supposé que la vitesse $v$ du déplacement du front est connue et dépend seulement de la courbure $\kappa$ de ce dernier. Pour les problèmes de la $\mathcal{A}$-étalabilité, cette vitesse est générée par la dynamique interne du système. Il serait alors intéressant de pouvoir calculer cette vitesse d'évolution du front à partir de la dynamique du système dans le cas où ce dernier est $\mathcal{A}$-étalable ; mais ce problème est très difficile.

\section{5. Étalabilité et contrôle}

Nous allons nous intéresser, dans cette partie, aux systèmes qui ne sont pas, par nature, étalables au sens des aires mais qui peuvent devenir étalables sous l'action d'un contrôle convenable. Forcer la résorbabilité d'un système par une action convenable est un problème symétrique. Considérons à titre d'exemple le système décrit par l'équation

$$
\left\{\begin{array}{l}
\frac{\partial z}{\partial t}(x, t)=b(x, t) z(x, t)+u(x, t) \quad t>0 ; x<a \\
z(x, 0)=z_{0}(x)=h(x+1)
\end{array}\right.
$$

où

et

$$
h(x)=\left\{\begin{array}{lll}
\exp \left(-\frac{1}{1-x}\right) & \text { si } & 0<x<1 \\
0 & \text { si } & 1 \leq x \leq 2 \\
\exp \left(\frac{1}{2-x}\right) & \text { si } & x>2
\end{array}\right.
$$

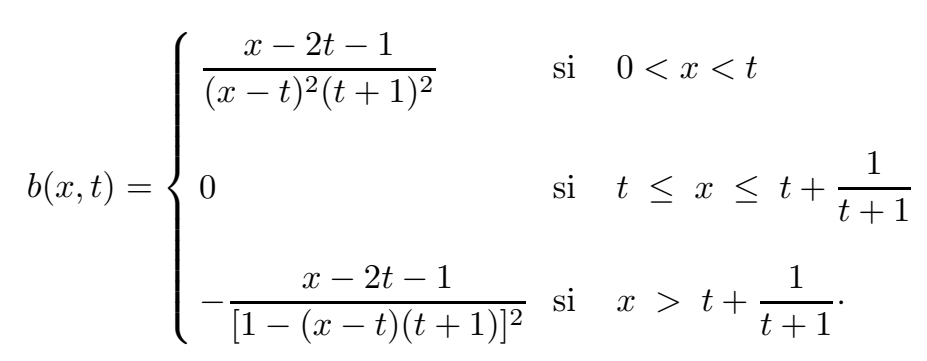


Intéressons-nous à la nulle-étalabilité au sens des aires avec $\omega_{t}=\{x \in \Omega \mid z(x, t)=0\}$, en partant du domaine initial $\omega_{0}=[0,1]$.

- Si le système est en évolution libre, $u=0$, alors pour $x>0$ et $t>0$, la solution est donnée par

$$
z(x, t)=\left\{\begin{array}{lll}
\exp \left(\frac{1}{(x-t)(t+1)}\right) & \text { si } & 0<x<t \\
0 & \text { si } & t \leq x \leq t+\frac{1}{t+1} \\
\exp \left(\frac{1}{1-(x-t)(t+1)}\right) & \text { si } & x>t+\frac{1}{t+1} .
\end{array}\right.
$$

Dans ce cas, il est facile de voir que $\omega_{t}=\left[t, t+\frac{1}{t+1}\right]$ et $\operatorname{mes}\left(\omega_{t}\right)=\frac{1}{1+t}$. Le système est alors $\mathcal{A}$-résorbable, voir figure 4 .

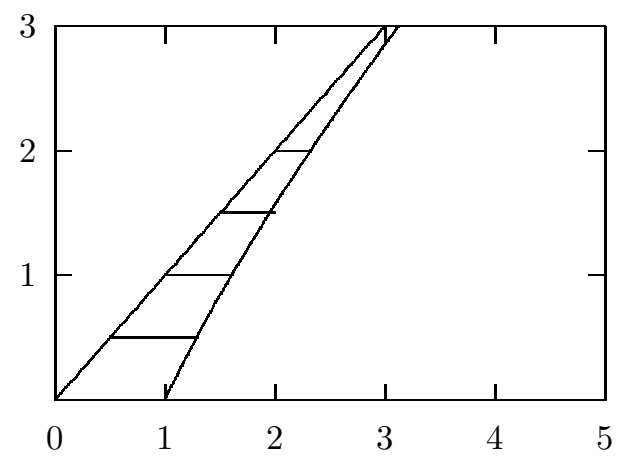

Figure 4 . Système autonome non $\mathcal{A}$-étalable.

- Si le système est excité par le contrôle

$$
\begin{aligned}
& u(x, t)= \\
& \left\{\begin{array}{l}
{\left[\frac{2(x+1)}{(2 x-t)^{2}}-\frac{x-2 t-1}{(x-t)^{2}(t+1)^{2}}\right] \exp \left(\frac{1}{\frac{x-t / 2}{t / 2+1}}\right) \text { si } 0<x<t / 2} \\
0 \quad \text { si } t / 2 \leq x \leq t+1 \\
{\left[-\frac{x+1}{2(t-x+1)^{2}}-\frac{x-2 t-1}{[1-(x-t)(t+1)]^{2}}\right] \exp \left(\frac{1}{1-\frac{x-t / 2}{t / 2+1}}\right) \text { si } x>t+1}
\end{array}\right.
\end{aligned}
$$


la solution est donnée par

$$
z_{1}(x, t)= \begin{cases}\exp \left(\frac{1}{\left.\frac{x-t / 2}{t / 2+1}\right)}\right. \text { si } & 0<x<t / 2 \\ 0 & \text { si } \quad t / 2 \leq x \leq t+1 \\ \exp \left(\frac{1}{1-\frac{x-t / 2}{t / 2+1}}\right) \text { si } & x>t+1\end{cases}
$$

et dans ce cas $\omega_{t}=\omega_{t}^{u}=\left[\frac{t}{2}, t+1\right]$ et $\operatorname{mes}\left(\omega_{t}^{u}\right)=\frac{t}{2}+1$. Le système contrôlé est alors $\mathcal{A}$-étalable sous l'effet du contrôle $u(x, t)$ donné en (3.35), voir figure 5 .

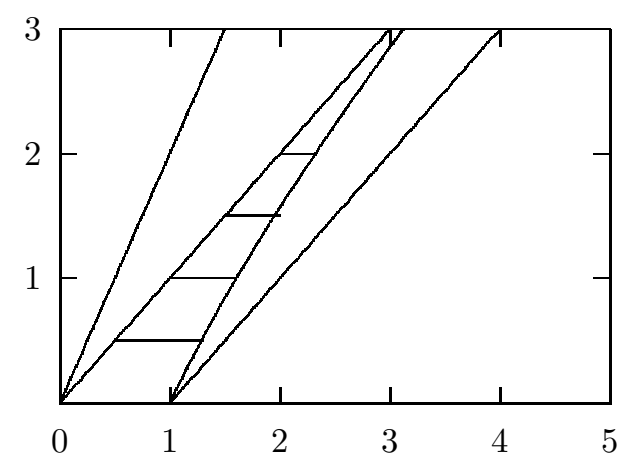

Figure 5. Système excité $\mathcal{A}$-étalable.

Cet exemple montre qu'un système peut devenir étalable au sens des aires sous l'action d'un contrôle convenable, que nous définirons comme étant un contrôle étaleur au de l'inclusion (s'il y a étalabilité au sens de l'inclusion) ou contrôle étaleur au des aires (s'il y a étalabilité au sens des aires).

Définition 3.15. On dira que le contrôle $u$ est un contrôle étaleur au sens des aires (ou contrôle $\mathcal{A}$-étaleur) si le système $(S)$ excité par $u$ est étalable au sens des aires.

De cette définition, il résulte évidemment que tout contrôle étaleur au sens de l'inclusion [20,56], l'est également au sens des aires mais la réciproque n'est pas vraie. Dans la suite et sans perte de généralité, on ne considérera que le cas de la nulle-étabilité en ne s'intéressant qu'aux sous-domaines $\omega_{t}$ de $\Omega$ dans lesquels l'état du système est nul. Nous noterons, pour simplifier,

$$
\{z(t, v)=f\}=\{x \in \Omega \mid z(x, t, v)=f(x)\}
$$

Le problème du contrôle $\mathcal{A}$-étaleur (ou étaleur) consiste à déterminer un contrôle $u$ qui rende un système $\mathcal{A}$-étalable (ou étalable) ; c'est-à-dire qui assure la croissance des mesures des domaines $\omega_{t}$. Notons que la résolution du problème du contrôle faiblement étaleur a été possible en introduisant des fonctions mesurables $\tau$ qui permettent de caractériser les étalements $\left(\sigma_{t}\right)$ [92].

Nous commençons par donner une caractérisation des contrôles étaleurs au sens des aires. Comme cela a été montré, les familles de transformations $(F(., s, t))_{s \geq t}$ adaptées à un système dynamique ont un rôle important dans la caractérisation des systèmes étalables au sens des aires. Elles s'avèrent aussi d'une grande utilité pour la caractérisation et la détermination des contrôles étaleurs au sens des aires. Nous avons la définition suivante. 
Définition 3.16. Nous dirons que la famille de transformations $(F(., s, t))_{s \geq t}$ est adaptable à l'étalabilité du système $(S)$ (ou tout simplement adaptable au système $(S)$ ) s'il existe un contrôle $u$ tel que la famille $(F(., s, t))_{s \geq t}$ soit adaptée au système $(S)$ excité par $u$. Nous dirons que $u$ est associé à $(F(., s, t))_{s \geq t}$.

Comme conséquence directe de la proposition 3.12 sur la caractérisation des systèmes étalables au sens des aires et de la définition 3.16 , nous avons le résultat suivant.

Proposition 3.17. Un contrôle u est un contrôle étaleur au sens des aires s'il est associé à une famille de transformations $(F(., s, t))_{s \geq t}$ satisfaisant

$$
\operatorname{mes}\left(F\left(\omega_{t}, s, t\right)\right) \geq \operatorname{mes}\left(\omega_{t}\right), \quad \forall s \geq t \geq t_{0} .
$$

Si la famille de transformations $(F(., s, t))_{s \geq t}$ de $\Omega$ est une famille de difféomorphismes de $\Omega$, cette condition est équivalente à

$$
\int_{\omega_{t}}[|\mathcal{J} F(x, s, t)|-1] \mathrm{d} x \geq 0 ; \quad \forall s, t, s \geq t \geq t_{0}
$$

ou encore si $F\left(\omega_{t}, s, t\right)=\omega_{s} \forall s, t, s \geq t \geq t_{0}$

$$
\frac{\partial}{\partial t} \int_{\omega_{t_{0}}}\left|\mathcal{J} F\left(x, t, t_{0}\right)\right| \mathrm{d} x \geq 0 ; \quad \forall t \geq t_{0}
$$

où $\mathcal{J} F(x, s, t)$ est le jacobien de la transformation $F(x, s, t)$ au point $x$.

\section{Remarque 3.18.}

- Si l'on choisit $F(., s, t)=I_{d_{\Omega}}$, le contrôle $u$ sera un contrôle étaleur au sens de l'inclusion.

- Pour une famille adaptable à $(S)$, le contrôle associé $u$ n'est pas forcément unique.

Il est alors intéressant de caractériser les contrôles associés à une famille de transformations adaptable à un système donné. On a le résultat suivant.

Théorème 3.19. Soient $(F(., s, t))_{s \geq t}$ une famille de transformations de $\Omega$ adaptable au système $(S)$ et $v$ un contrôle donné. Alors $v$ est associé à $(F(., s, t))_{s \geq t}$ s'il existe une fonction $\xi$ solution de l'équation

$$
\Theta(\xi, v)=0
$$

où

$$
\Theta(\xi, v)=\int_{t}^{T} z^{2}[F(x, s, t), s, v] \mathrm{d} s-\xi(x, t) z(x, t, v) .
$$

La preuve est évidente d'après la caractérisation des familles adaptées à un système (Prop. 3.11). Comme conséquence du théorème 3.19 , on a une caractérisation des familles adaptables à un système. En effet $(F(., s, t))_{s \geq t}$ est adaptable au système $(S)$ s'il existe une fonction $\xi$ et un contrôle $v$ solutions de l'équation (3.40).

Considérons maintenant le problème de contrôle étaleur pour le système représenté par l'équation d'état

$$
\left\{\begin{array}{l}
\dot{z}=A z+B v \quad ; t \in I=] t_{0}, T[ \\
z\left(t_{0}\right)=z_{0} \in D(A)
\end{array}\right.
$$


dans lequel on suppose que $A$ est un opérateur linéaire qui génère un semi-groupe fortement continu $(S(t))_{t \geq 0}$ sur l'espace d'état $Z=L^{2}(\Omega), B$ est un opérateur linéaire de l'espace des contrôles $\mathcal{U}$ dans l'espace d'état $Z$. La solution de (3.41) est alors donnée par

$$
z(., t, v)=S\left(t-t_{0}\right) z_{0}(.)+\int_{t_{0}}^{t} S(t-s) B v(s) \mathrm{d} s .
$$

On introduit l'opérateur $H: \mathcal{V} \longrightarrow L^{2}(\Omega \times I)$ avec $\mathcal{V}=L^{2}\left(t_{0}, T, \mathcal{U}\right)$, défini par

$$
H v(., t)=\int_{t_{0}}^{t} S(t-s) B v(s) \mathrm{d} s
$$

ainsi (3.42) s'écrit

$$
z(., t, v)=S\left(t-t_{0}\right) z_{0}+H v(., t) .
$$

Nous considérons le cas de la nulle-étalabilité, $\omega_{t}^{v}=\{z(t, v)=0\}, t \in I, v \in \mathcal{V}$, et nous supposons que $\operatorname{mes}\left(\omega_{0}\right)=\operatorname{mes}\left(\left\{z_{0}=0\right\}\right) \neq 0$. Comme la notion d'étalabilité au sens des aires ne concerne que la mesure des domaines $\omega_{t}^{v}$, on pose de façon naturelle le problème de contrôle comme suit

$$
(P)\left\{\begin{array}{l}
\text { Déterminer } v \in \mathcal{V} \text { satisfaisant (3.42) } \\
\text { tel que } \\
\operatorname{mes}\left(\omega_{t}^{v}\right) \text { soit croissante. }
\end{array}\right.
$$

Le fait de n'imposer que la croissance des mesures des domaines $\left(\omega_{t}^{v}\right)$, sans aucune condition sur la manière d'évolution des sous-domaines $\left(\omega_{t}\right)_{t \in I}$, laisse beaucoup de possibilités. Il suffit alors de choisir une famille de transformations $(F(., s, t))_{s \geq t}$ convenable et de déterminer un contrôle qui lui est associé.

La résolution du problème de contrôle se fera alors en deux étapes

1. choisir une famille de transformations $(F(., s, t))_{s \geq t}$ convenable (satisfaisant par exemple (3.37) ou (3.38)) ;

2. déterminer un contrôle associé à $(F(., s, t))_{s \geq t}$.

Si maintenant la famille de transformations $(F(., s, t))_{s \geq t}$ est donnée, alors un contrôle existe si cette famille $(F(., s, t))_{s \geq t}$ est adaptable au système (3.41). Nous commençons par chercher des conditions suffisantes pour que la famille $(F(., s, t))_{s \geq t}$ soit adaptable à $(3.41)$; nous donnerons ensuite un contrôle associé.

Reprenons le système linéaire donné par (3.41) et supposons en plus que l'opérateur $B$ est borné et que $\operatorname{Im}(H) \subset L^{\infty}(\Omega \times I)$. La famille $(F(., s, t))_{s \geq t}$ est adaptable au système (3.41) s'il existe un couple $(\xi, v)$ solution de l'équation (3.40). Nous allons transformer le problème de la recherche d'une solution de l'équation (3.40) en la recherche d'un point fixe d'un certain opérateur à définir. Pour cela, posons

$$
\left(H_{1} v\right)(., t)=(H v)\left(F\left(., t, t_{0}\right), t\right)
$$

et

$$
z_{1}(., t, v)=z\left[F\left(., t, t_{0}\right), t, v\right]
$$

alors on a

$$
z_{1}(., t, v)=S\left(t-t_{0}\right) z_{0}\left[F\left(., t, t_{0}\right)\right]+\left(H_{1} v\right)
$$

Pour un contrôle $v_{0}$ fixé dans $\mathcal{V}$, on a

$$
z_{1}(., t, v)-z_{1}\left(., t, v_{0}\right)=\left(H_{1} v\right)-\left(H_{1} v_{0}\right)
$$


ce qui donne, en notant $z_{1}(v)=z_{1}(., t, v)$

$$
z_{1}(v)=z_{1}\left(v_{0}\right)+H_{1}\left(v-v_{0}\right) .
$$

On suppose que $(F(., s, t))_{s \geq t}$ est une famille de difféomorphismes de $\Omega$ satisfaisant la relation

$$
F\left(x, s, t_{0}\right)=F(x, s, t) \circ F\left(x, t, t_{0}\right) \quad \forall x \in \Omega \text { et } \forall s \geq t .
$$

Alors dans ce cas, l'équation (3.40) est équivalente à

$$
\Theta_{1}(\xi, v)=0
$$

où

$$
\Theta_{1}(\xi, v)=\int_{t}^{T} z^{2}\left[F\left(x, s, t_{0}\right), s, v\right] \mathrm{d} s-\xi(x, t) z\left(F\left(x, t, t_{0}\right), t, v\right) .
$$

En effet il est évident que si (3.40) est satisfaite alors (3.49) l'est aussi. Réciproquement montrons que si (3.49) est satisfaite alors la famille $(F(., s, t))_{s \geq t}$ est adaptable au système. Si $x_{t} \in \omega_{t}$, alors $z\left(x_{t}, t\right)=0$ et d'après les hypothèses sur $(F(., s, t))_{s \geq t}$, il existe $x_{0} \in \omega_{0}$ tel que $F\left(x_{0}, t, t_{0}\right)=x_{t}$. La relation $(3.48)$ donne $F\left(x_{0}, s, t_{0}\right)=$ $F\left(x_{t}, s, t\right)$. Donc Si l'équation (3.49) est satisfaite alors $z\left(F\left(x_{0}, s, t_{0}\right), s\right)=0 \forall s \geq t$ et par suite $z\left(F\left(x_{t}, s, t\right), s\right)=$ 0 . Donc $F\left(\omega_{t}, s, t\right) \subset \omega_{s} \forall s \geq t$. Ce qui montre que (3.40) est satisfaite.

Dans la suite on notera indifféremment $\Theta_{1}(\xi, v)$ ou $\Theta(\xi, v)$. Posons $w=v-v_{0}$ et avec (3.47) et (3.49) on obtient

$$
\Theta(\xi, v)=0 \Leftrightarrow \ell_{1}(\xi, w)=-\left(q_{1}+c_{1}\right)(\xi, w)
$$

où

$$
\begin{aligned}
& q_{1}=\int_{t}^{T}\left(H_{1} w\right)^{2}(x, s) \mathrm{d} s-\xi(x, t)\left(H_{1} w\right)(x, t) \\
& \ell_{1}=2 \int_{t}^{T} z_{1}\left(x, s, v_{0}\right)\left(H_{1} w\right)(x, s) \mathrm{d} s-\xi(x, t) z_{1}\left(x, t, v_{0}\right) \\
& c_{1}=\int_{t}^{T} z_{1}^{2}\left(x, s, v_{0}\right) \mathrm{d} s .
\end{aligned}
$$

Si l'opérateur linéaire $\ell_{1} \ell_{1}^{*}$ est inversible dans $L^{2}(\Omega \times I) \times \mathcal{V}$, en notant $\ell_{1}^{\dagger}$ le pseudo-inverse de $\ell_{1}$, alors $(\xi, v)$ est une solution de (3.49) si et seulement si $(\xi, w)$ est solution de l'équation

$$
(\xi, w)=-\ell_{1}^{\dagger}\left(q_{1}+c_{1}\right)(\xi, w)
$$

Posons

$$
G(\xi, w)=-\ell_{1}^{\dagger}\left(q_{1}+c_{1}\right)(\xi, w)
$$

Ainsi la détermination d'une solution $(\xi, v)$ de $(3.49)$ revient à trouver un point fixe de $G$. Soient maintenant $(\xi, \omega),(\bar{\xi}, \bar{\omega}) \in B(0, m) \subset L^{2}(\Omega \times I) \times \mathcal{V}$ où $B(0, m)$ est la boule de centre 0 et de rayon $m$, alors si $\ell_{1}^{\dagger}$ est borné, on a

$$
\| G(\xi, \omega)-G\left(\bar{\xi},(\bar{\omega})\|\leq\| \ell_{1}^{\dagger}\|\cdot\| q_{1}(\xi, \omega)-q_{1}(\bar{\xi}, \bar{\omega}) \|\right.
$$

Posons $k=\left\|\ell_{1}^{\dagger}\right\| ; \beta=\|b\|$ et $\zeta=\left\|c_{1}\right\|$ où $b$ est l'opérateur bilinéaire symétrique défini sur $L^{2}(\Omega \times I) \times \mathcal{V}$ par $q_{1}(\xi, \omega)=b(\xi, \omega) .(\xi, \omega)$. Alors

$$
\| G(\xi, \omega)-G(\bar{\xi},(\bar{\omega})\|\leq k \beta\|(\xi, \omega)+(\bar{\xi}, \bar{\omega})\|.\|(\xi, \omega)-(\bar{\xi}, \bar{\omega}) \|
$$


ce qui donne

$$
\| G(\xi, \omega)-G(\bar{\xi},(\bar{\omega})\|\leq 2 k \beta m\|(\xi, \omega)-(\bar{\xi}, \bar{\omega}) \| .
$$

Il suffit alors maintenant de choisir $m$ tel que

$$
\left\{\begin{array}{l}
2 m \beta k<1 \\
G(B(0, m)) \subset B(0, m)
\end{array}\right.
$$

et dans ce cas $G$ admettra un point fixe $\left(\xi^{*}, \omega^{*}\right)$ dans $B(0, m)$. Le choix de $m$ dépend du choix de $v_{0}$, on a le résultat suivant.

Proposition 3.20. S'il existe un contrôle $v_{0} \in \mathcal{V}$ tel que,

$$
\mathcal{H}\left\{\begin{array}{l}
\left\|h z_{1}\left(v_{0}\right)\right\|^{2}+\left\|H_{1}^{*}\left(2 z_{1}\left(v_{0} r_{h}\right)\right)\right\|^{2} \geq \mu h^{2} \\
r_{h}(x, t)=\int_{t_{0}}^{t} h(x, s) \mathrm{d} s \quad((x, t) \in \Omega \times I) \\
\forall h \in L^{2}(\Omega \times I)
\end{array}\right.
$$

alors l'opérateur linéaire $\ell_{1} \ell_{1}^{*}$ est inversible dans $L^{2}(\Omega \times I) \times \mathcal{V}$ et son pseudo-inverse, donné par

$$
\ell_{1}^{\dagger}=\ell_{1}^{*}\left(\ell_{1} \ell_{1}^{*}\right)^{-1}
$$

est borné. Si de plus on a

$$
4 k^{2} \beta \zeta<1
$$

alors la condition (3.53) est vérifiée pour tout

$$
m \in] \frac{1-\sqrt{1-4 k^{2} \beta \zeta}}{2 k \beta}, \frac{1}{2 k \beta}[.
$$

Pour la preuve, voir $[28,30,56]$.

Corollaire 3.21. Si un contrôle v vérifie l'hypothèse $\mathcal{H}$ et (3.55) alors

(i) la famille $(F(., s, t))_{s \geq t}$ est adaptable à $(S)$;

(ii) l'opérateur $G$ admet un point fixe $\left(\xi^{*}, \omega^{*}\right)$ et $v^{*}=v_{0}+\omega^{*}$ est un contrôle associé à la famille de transformations $(F(., s, t))$.

Ceci résulte immédiatement de la proposition (3.20) et de ce qui précède. Ainsi pour déterminer un contrôle étaleur au sens des aires, il suffit de choisir d'avance une famille de transformations convenable et de trouver un contrôle qui lui sera associé. Pour un tel contrôle, la famille de transformations $(F(., s, t))_{s \geq t}$ est adaptable à l'étalabilité du système $(S)$. La caractérisation des familles adaptables à un système conduit au résultat d'existence suivant.

Corollaire 3.22. Si la famille de transformations $(F(., s, t))_{s \geq t}$ est une famille de difféomorphismes de $\Omega$ satisfaisant (3.48) et (3.38) et s'il existe un contrôle $u_{0}$ vérifiant l'hypothèse $\mathcal{H}$ et (3.55), alors le problème $(P)$ admet au moins une solution. 
Remarque 3.23. Si on prend $F(., s, t)=I d_{\Omega}$, on retrouve bien le problème de contrôle étaleur au sens de l'inclusion [56]. En effet le problème devient

$$
(P 1)\left\{\begin{array}{l}
\text { Déterminer } v \in \mathcal{V} \text { satisfaisant }(3.42) \\
\text { tel que } \\
\omega_{t} \subset \omega_{s} \text { pour } s \geq t
\end{array}\right.
$$

Comme dans ce cas $|\mathcal{J} F(x, s, t)|=1$ sur $\Omega$, alors la condition (3.38) est bien vérifiée. On retrouve le même résultat que dans $[20,56]$ en résolvant l'équation (3.40).

Nous pouvons considérer le problème du contrôle étaleur en s'intéressant au suivi des interfaces. Pour cela, considérons toujours $\omega_{t}=\{x \in \Omega \mid z(x, t)=p(x)\}$ où $p$ est donné et $\Gamma(t)=\partial \omega_{t}$. Nous pouvons alors considérer le problème suivant.

$$
(P 2)\left\{\begin{array}{l}
\text { Déterminer un contrôle étaleur } u \in \mathcal{V} \\
\text { tel que } \\
\text { Le front } \Gamma(t) \text { évolue avec une vitesse } v \text { donnée. }
\end{array}\right.
$$

Ici il s'agit de déterminer un contrôle $u$ qui assure l'étalabilité au sens des aires avec une certaine vitesse $v$ d'évolution du front $\Gamma(t)$. Il n'est pas évident que ce problème admette une solution. On peut plus facilement déterminer des contrôles étaleurs sans toutefois pouvoir contrôler la vitesse de l'étalement. Illustrons cela en considérant l'exemple donné par l'équation

$$
\begin{cases}\frac{\partial z}{\partial t}+\frac{\partial z}{\partial x}=\delta(x-b) u(t) & x>0 ; t>0 \\ z(0, t)=0 & t>0 \\ z(x, 0)=z_{0}(x) & x>0\end{cases}
$$

où $b>0$ et intéressons-nous à sa nulle-étalabilité au sens des aires à partir de $\omega_{0}=\{b\}$. Nous avons déjà vu que, dans le cas où ce système est autonome, on a

$$
\omega_{t}=[0, t[\cup\{b+t\} \quad t>0 .
$$

Le système est alors étalable au sens des aires à cause de la condition homogène en $x=0$. La solution de ce système excité par un contrôle ponctuel localisé au point $b$ est donnée par

$$
z(x, t, u)= \begin{cases}z_{0}(x-t)+u(b+t-x) & \text { si } x \in[b, b+t], \quad x \geq t \\ z_{0}(x-t) & \text { si } x \notin[b, b+t], \quad x \geq t \\ u(b+t-x) & \text { si } x \in[b, b+t], \quad x<t \\ 0 & \text { si } x \notin[b, b+t], \quad x<t\end{cases}
$$

ainsi, pour $b>T$, si nous considèrons le contrôle

$$
u(t)=-z_{0}(b-t) \quad ; \quad 0 \leq t<T
$$

alors (3.58) conduit à

$$
\omega_{t}^{u}=[0, t[\cup[b, b+t[
$$

Le système est étalable au sens des aires "à partir" du point $b$ mais la vitesse d'évolution du point $b+t$ est constante et égale à 1 . Le contrôle choisi en (3.59) assure l'étalabilité au sens des aires mais ne permet pas de choisir la vitesse d'étalement. 
Le problème $(P 2)$ peut être traité en utilisant une famille de transformations $(F(., s, t))_{s \geq t}$ adaptable à l'évolution du système. La résolution se fait alors en considérant les deux étapes données précédemment. Pour la détermination de la famille $(F(., s, t))_{s>t}$ à partir du champ de vecteur de vitesse du front, c'est plus difficile car on ne dispose pas de résultats explicites. Pour la détermination d'une telle famille, voir [90]. Mais dans certains cas particuliers, il est possible de déterminer une telle famille de transformations, et par voie de conséquence le contrôle convenable. Un exemple est donné dans [20].

\section{RÉFÉRENCES}

[1] L. Afifi, Analyse régionale : systèmes distribués discrets - Source et détection, Thèse de Doctorat d'État. Kenitra, Maroc (1994).

[2] L. Afifi et A. El Jai, Strategic sensors and spy sensors. J. Appl. Math. Comput. Sci. 4 (1994) 553-573.

[3] L. Afifi et A. El Jai, Observabilité des systèmes distribués discrets dans un tube. J. Marocain d'Automatique d'Informatique et de Traitement de Signal II (1994) 1-45.

[4] L. Afifi et A. El Jai, Spy sensors and detection. Internat. J. Syst. Sci. 25 (1995) 1447-1463.

[5] L. Afifi, A. El Jai et M. Merry, Détection dans un tube, Rapport No. 15/98. LTS, Université de Perpignan, France (1998).

[6] L. Afifi, A. Chafiai et A. El Jai, Compensation spatiale en temps fini dans les Systèmes Distribués, Rapport No. 15/98. LTS, Université de Perpignan, France (1998).

[7] L. Afifi, A. Chafiai et A. El Jai, Regionally efficient and strategic actuators. Internat. J. Syst. Sci. (à paraître).

[8] L. Afifi, A. El Jai et M. Merry, Detection and sources reconstruction in a tube. Internat. J. Syst. Sci. 31 (2000) 149-159.

[9] J.P. Aubin, Viability theory, Systems and Control: Foundations and Applications. Birkhauser (1991).

[10] S. Angenent, Shrinking Doughnuts, dans Proc. of Nonlinear Diffusion Equations and Their Equilibrium States, Vol. 3, édité par N.G. Lloyd et al. Birkhauser, Boston, MA (1992).

[11] G. Barles, Remark on a flame propagation model. Rapport INRIA, 464 (1985).

[12] G. Barles, H.M. Soner et P.E. Souganidis, Front propagation and phase field theory. SIAM J. Control Optim. 31 (1993) 439-469.

[13] G. Barles et P.E. Souganidis, A new approach to front propagation problems: Theory and applications. Arch. Rational Anal. 141 (1998) 237-296.

[14] G. Barles, An Introduction to recent developments in front propagation problems, dans Écoles CEA-EDF-INRIA. Cours : suivi d'interfaces. INRIA Rocquencourt, France (1999).

[15] A.V. Balakrishnan, Applied functional analysis. Springer-Verlag (1976).

[16] A. Belfekih, Global approach to spreadability (2001).

[17] E. Beltrami, Mathematics for Dynamic Modeling. Academic press (1987).

[18] M.S. Berger, Nonlinearity and functional Analysis. Academic Press, Lectures on Nonlinear Problems in Math. Anal.

[19] A. Berger et C. Tricot, Simple Climate Models Climatic Change and Impact: A General Introduction, dans Proc. of the European School of Climatology and Natural Hazards Course. Florence, 11-18 september 1988, édité par R. Fantechi et al. (1988).

[20] A. Bernoussi, Vers un nouveau concept dans les systèmes distribués : l'étalabilité. HDR, Université de Perpignan (2001).

[21] A. Bernoussi et A. El jai, Spreadability and evolving interfaces. AMIF: Applied Mathematics For Industrial Flow Problems. An ESF International Conference, Sant Feliu de Guixols (Barcelona), 1-3 October 1998. Theme 3: Fronts, interfaces and free boundaries (1998).

[22] A. Bernoussi et A. El Jai, À propos de l'étalabilité, Rapport No. 10/98. LTS, Université de Perpignan (1998).

[23] A. Bernoussi, Nouvelle approche de l'étalabilité : A-étalabilité, JANO 5, 28/30 Avril. Kenitra, Maroc (1998).

[24] A. Bernoussi, Spreadability and spray control, dans Journée thématique : Modélisation et contrôle des systèmes, 30 mars 1999. Perpignan, France (1999).

[25] A. Bernoussi et A. El Jai, Spreadability, wavefront and evolving interfaces, Rapport 4/99. LTS, France (1999) 1-36.

[26] A. Bernoussi et A. El Jai, New approach of spreadability, Rapport, No. 5/99. LTS, France (1999) 1-22.

[27] A. Bernoussi et A. El jai, Distributed Parameters Systems Spreadability and evolving interfaces, MTNS 2000, 19-23 june. Perpignan, France (2000).

[28] A. Bernoussi et A. El Jai, New Approach of Spreadability. J. Math. Comput. Modelling 31 (2000) 93-109.

[29] A. Bernoussi et A. El Jai, Étalabilité au sens des aires, JANO6, 8-11 mars, 2000. Casablanca, Maroc (2000).

[30] A. Bernoussi, A. El Jai et A.J. Pritchard, Spreadability and evolving interfaces. Int. J. Syst. Sci. (2001) 1-16.

[31] Ah. Bernoussi, Étalabilité et contrôle étaleur, Thèse de Doctorat. Université de Perpignan (1999).

[32] Ah. Bernoussi et D. Ucinski, Weak Spray Control, dans Second Workshop on Analysis and Optimization of complex environmental systems. Zielona Gora, Poland (1998).

[33] Ah. Bernoussi, A. El Jai et D. Ucinski, Modified Weak Spray Control. Internat. J. Control (proposé).

[34] L. Berrahmoune, Controlability of dampted flexible systems, dans Fifth IFAC Symposium on Control of Distributed Parameter Systems. Perpignan, France (1989). 
[35] L. Berrahmoune, Actionneurs et capteurs dans la contrôlabilité et la stabilité de systèmes distribués flexibles, Thèse de Doctorat d'État. Faculté des Sciences de Rabat, Maroc (1990).

[36] L. Berrahmoune et A. El Jai, On Hilbert uniqueness method: A semi-group approach, dans Fifth IFAC Symposium on Control of Distributed Parameter Systems. Perpignan, France (1989).

[37] K.A. Brakke, The motion of a surface by its Mean Curvature. Princeton University Press, Princeton (1978).

[38] B. Chopard et M. Droz, Cellular automata modelling of physical systems. Collection Alea-Saclay (1998).

[39] R.F. Curtain et A.J. Pritchard, Infinite Dimensional Linear Systems Theory. Springer, Lectures Notes in Control and Inform. Sci. 8 (1978).

[40] Xu-Yan Chen, Dynamics of interfaces in reaction diffusion systems. Hirochima Math. J. 21 (1991) 47-83

[41] D.L. Chopp, Numerical Computation of Self-Similar Solutions for Mean Curvature Flow. J. Exper. Math. 3 (1994) 1-15.

[42] R.L. Colasanti et J.P. Grime, Resource Dynamics and Vegetation processes: A deterministic model using two-dimensional cellular automata. Functional Ecology 7 (1993) 169-176.

[43] R.F. Dautray et J.-L. Lions, Analyse mathématique et calcul numérique pour les sciences et les techniques, Série scientifique, Tome 3. Masson (1984).

[44] J.I. Diaz et J.-L. Lions, Mathematics Climate and Environment. Masson, Res. Notes in Appl. Math. RMA 27 (1993).

[45] M.C. Delfour et S.K. Mitter, Controlability and observability for infinite dimensional systems. SIAM J. Control. 10 (1972) 329-333.

[46] S. Dolecki, Observability for the one dimensional heat equation. Stud. Math. 48 (1973) 291-305.

[47] S. Dolecki et D. Russel, A general theory of observation and control. SIAM J. Control 15 (1977) 185-220.

[48] A. El Jai, Distributed Systems Analysis via Actuators and Sensors. Internat. J. Sensors and Actuators 29 (1991) 1-11.

[49] A. El Jai, Systems analysis approach to vegetation dynamics, Plenary lecture, dans 2nd International Conference on Differential Equations. Marrakech (1995).

[50] A. El Jai, Spreadability of DPS, MTNS (Mathematical Theory of Networks and Systems). St. Louis Missouri, USA (1996).

[51] A. El Jai, Nouvelle approche pour la modélisation des systèmes en expansion spatiales : dynamique de végétation, Tendances nouvelles en modélisation pour l'environnement. Elsevier (1997) 439-445.

[52] A. El Jai et L. Berrahmoune, Localisation d'actionneurs zones pour la contrôlabilité de systèmes paraboliques. $C$. $R$. Acad. Sci. 297 (1983) 647-650.

[53] A. El Jai et L. Berrahmoune, Localisation d'actionneurs ponctuels pour la contrôlabilité de systèmes paraboliques. $C$. $R$. Acad. Sci. 298 (1983) 47-50.

[54] A. El Jai et L. Berrahmoune, Localisation d'actionneurs frontières pour la contrôlabilité de systèmes paraboliques. $C$. $R$. Acad. Sci. 298 (1984) 177-180.

[55] A. El Jai et S. El Yacoubi, Automates cellulaires et étalabilité, Report. Université de Perpignan (1996).

[56] A. El Jai et K. Kassara, Spreadable distributed systems. Math. Comput. Modelling 20 (1994) 47-64.

[57] A. El Jai et K. Kassara, Spreadability of transport systems. Internat. J. Syst. Sci. 27 (1996) 681-688.

[58] A. El Jai et A.J. Pritchard, Distributed parameter Systems Analysis via sensors and actuators. J. Wiley, Texts in Appl. Math. (1988).

[59] A. El Jai et A.J. Pritchard, Regional controllability of Distributed Systems, dans Int. INRIA-IFAC Conf. on Optimization and Analysis of Systems. Sophia Antipolis (1992).

[60] A. El Jai, S. El Yacoubi et J. Karrakchou, Spreadability and spray actuators. Appl. Math. Comput. Sci. 2 (1998).

[61] A. El Jai, K. Kassara et O. Cabrera, Spray control. Internat. J. Control 68 (1997) 709-730.

[62] A. El Jai, M.C. Simon et E. Zerrik, Regional observability and sensors structures. Internat. J. Sensors and Actuators 39 (1993) 301-313.

[63] A. El Jai, M.C. Simon et E. Zerrik, Numerical approach for regional observation problem. Application to a thermal process, dans 14th IMACS World Congress Atlanta, Georgie, USA (1994).

[64] A. El Jai, M. Fournier, K. Kassara et B. Noumare, Vegetation Dynamics: A deterministic modelling approach using the concept of spreadability. Theoret. Appl. Climatology J. 52 (1995) 241-249.

[65] A. El Jai, M.C. Simon, E. Zerrik et A.J. Pritchard, Regional controllability of distributed systems. Internat. J. Control 62 (1995) 1351-1365.

[66] A. El Jai, E. Zerrik, M.C. Simon et M. Amouroux, Regional observation of a thermal process. IEEE Trans. Automat. Control 40 (1995)

[67] A. El Jai, O. Cabrera, M. Fournier et Y. Maurissen, A prediction-correction alternative for modelling spatio-temporal systems. Application to an ecological problem, Ecological Modelling. Ecomod195 (1998).

[68] S. El Yacoubi et J. Karrakchou, DPS Modelling via cellular automata. Application to delay systems, dans Conference on Numerical Analysis and Optimization. Kenitra, Morocco (1998).

[69] S. El Yacoubi et A. El Jai, DPS Modelling and cellular automata. Math. Comput. Modelling (à paraître).

[70] L.C. Evans, H.M. Soner et P.E. Souganidis, Phase transitions and generalized motion by mean curvature. I. J. Diff. Geom. 33 (1992) 635-681.

[71] S. Goto, Generalized motion of hypersurfaces whose growth speed depends superlinearly of the curvature tensor. Differential Int. Equations 7 (1994) 323-343. 
[72] Y. Giga et M.H. Sato, Generalized interface evolution with Neumann boundary condition. Proc. Japan Acad. Ser. A 67 (1991) 263-266.

[73] T.Y. Hou, An Numerical solutions to free boundary problems. Écoles CEA-EDF-INRIA. Cours : suivie d'interfaces. INRIA Rocquencourt, France (1999).

[74] K. Kassara, Étalabilité des systèmes distribués, Thèse de Doctorat d'État. EMI, Rabat, Maroc (1999).

[75] A. Kolmogoroff, I. Petrovsky et N. Piscounoff, Étude de l'équation de la diffusion avec croissance de la quantité et son application à un problème biologique. Moscou Univ. Bull. Math. 1 (1937) 1-25.

[76] I. Lasiecka et R. Trigiani, Control Problems for Systems Described by Partial Differential Equations and Application, dans Proc. of the IFIP-WG 7.2 Working Conference. Gainesville, Florida, February 3-6 (1986). Springer-Verlag, Lecture Notes in Control and Inform. Sci. (1987).

[77] J.-L. Lions, Contrôlabilité exacte, Perturbation et Stabilisation des systèmes distribués, RMA 8 et 9. Masson, Paris (1988).

[78] J.-L. Lions, Some methods in the mathematical analysis of systems and their control. Science Press. Beijing, China (1981). Gordon and breach, Science Publishers, Inc. New York (1981).

[79] A.M. Micheletti, Perturbazione dello spettro di un operatore ellitico di tipo variazionale, in relazione ad una variazione del campo. Ricerche di Mat. V.XXV (1976).

[80] D. Mollison, Spatial contact models for ecological and epidemic spread. J. Roy. Stat. Soc. B 39 (1997) 283-326.

[81] R.A. Monserud, N.M. Tchebakova et R. Leemans, Global Vegetation Change predicted by the modified Budyko Model. Climatic Change 25 (1993) 59-83.

[82] J.D. Murray, Mathematical Biology. Springer, Biomath. Texts (1993).

[83] M. Rachik, Quelques éléments sur l'analyse et le contrôle des systèmes distribués, Thèse de Doctorat d'État. Université de Rabat, Maroc (1995).

[84] S. Osher et J.A. Sethian, Fronts propagating with curvature-dependant speed: Algorithms based on Hamilton-Jacobi formulation. J. Comput. Phys. 79 (1988) 12-49.

[85] J.A. Sethian, Curvature and the evolution of fronts. Comm. Math. Phys. 101 (1985) 487-499.

[86] J.A. Sethian, Level set methods: Evolving interfaces in geometry, fluid mechanics, computer vision and material sciences. Campbridge Univresity Press, Cambridge Monogr. Appl. Comput. Math. (1996).

[87] J.A. Sethian, Level Set Methods and Fast Marching Methods. Evolving Interfaces in Computational Geometry, Fluid Mechanics, Computer Vision, and Materials Sciences. Cambridge University Press, Cambridge Monogr. Appl. Comput. Math. (1999).

[88] R. Sepulchre, M. Jankovic et P. Kokotovic, Constructive Nonlinear Control. Springer, Commun. and Control Engrg. (1997).

[89] H.M. Soner, Motion of a set by the curvature of its boundary. J. Differential Equations 101 (1993) 313-372.

[90] J. Sokolowski et J.P. Zolesio, Introduction to Shape Optimization, Shape Sensitivity Analysis. Springer-Verlag, Springer Ser. in Comput. Math. 16 (1992).

[91] A.M. Solomon et H.H. Shugart, Vegetation Dynamics and Global Change. IIASA. Chapman and Hall. Library of Congress Cataloging in Publication Data (1993).

[92] D. Ucinski et A. El Jai, On weak spreadability of DPS and its achievement via LQ Control Techniques. IMA J. Control and Inform. Sci. 14 (1997) 153-174.

[93] A.T. Winfree, The Geometry of Biological Time. Springer-Verlag, Berlin (1980).

[94] S. Wolfram, Cellular automata and complexity. Addison-Wesley Publishing Company, Sci. Math. Comput. (1994).

[95] A.N. Zaikin et A.M. Zhabotinski, Concentration wave propagation in two-dimensional liquid-phase self-oscillatting systems. Nature 225 (1970) 535-537.

[96] E.H. Zerrik, Regional Analysis of Distributed Parameter Systems, Thèse d'État. Faculté des Sciences de Rabat, Maroc (1993).

$[97]$ E.H. Zerrik, L. Badraoui et A. El Jai, Sensors and regional boundary state reconstruction of parabolic systems. Sensors and Actuators J. 75 (1999) 102-117.

[98] E.H. Zerrik, A. Boutoulout et A. El Jai, Actuators and regional boundary controllability of parabolic systems. Int. J. Syst. Sci. 31 (2000) 73-82.

[99] E.H. Zerrik, A. Kamal et A. Boutoulout, Regional gradient controllability of parabolic systems. Appl. Math. Comput. Sci. 9 (1999) 101-121. 